\title{
Montpellier
}

Étude archéologique d'un plafond décoré d'époque classique (9 rue de la Petite-Loge)

\section{Véronique Mathieu}

\section{(Q) OpenEdition Journals}

Édition électronique

URL : http://journals.openedition.org/adlfi/11933

ISSN : 2114-0502

Éditeur

Ministère de la culture

Référence électronique

Véronique Mathieu, «Montpellier », ADLFI. Archéologie de la France - Informations [En ligne], LanguedocRoussillon, mis en ligne le 01 mars 2004, consulté le 02 mai 2019. URL : http://

journals.openedition.org/adlfi/11933

Ce document a été généré automatiquement le 2 mai 2019.

(c) Ministère de la Culture et de la Communication, CNRS 


\section{Montpellier}

Étude archéologique d'un plafond décoré d'époque classique (9 rue de la Petite-Loge)

\section{Véronique Mathieu}

Identifiant de l'opération archéologique :

Date de l'opération : 1995 (SU)

Inventeur(s) : Mathieu Véronique (AFAN)

1 L'immeuble faisant l'objet de la présente intervention se trouve situé au 9 rue de la Petite-Loge à Montpellier (Hérault) (Fig. $\mathrm{n}^{\circ} 1$ : Plan de situation de Montpellier et ses environ vers 1731). Le plafond se localise au deuxième étage de la maison, dans sa partie la plus récente. Sa mise en communication avec le bâti initial est assurée au moyen d'un escalier sur trompe probablement mis en œuvre lors de l'agrandissement de la propriété.

2 En ce qui concerne la technique de relevé, nous avons procédé comme suit : après avoir établi le relevé en plan de la pièce concernée par l'opération à un niveau situé à environ un mètre du sol, nous avons repéré au fil à plomb le positionnement des poutres et des premières solives par rapport à ce plan fictif de relevé. Les autres éléments constitutifs du plafond furent ensuite repérés par rapport à ces poutres et premières solives [S3, S24, S45; (Fig. $n^{\circ} 3$ : Relevé d'état des lieux en plan du plafond)].

\section{Structure du plafond}

\section{Description}

Suivant la terminologie employée dans le Vocabulaire de l'Architecture [(Pérouse de Montclos, Jean-Marie. 1972.); Pérouse de Montclos, 1972 : tome I, p. 105], nous parlerons ici de plafond à solives apparentes (Fig. $\mathrm{n}^{\circ} 2$ : Vue générale sur la deuxième travée, de S27 à S32, vue depuis le nord-ouest). Il se compose de deux grosses poutres transversales, 
support de l'ensemble, ayant une section de $32 \mathrm{~cm}$ de hauteur pour environ $22 \mathrm{~cm}$ de largeur [ (Fig. $n^{\circ} 3$ : Relevé d'état des lieux en plan du plafond) et (Fig. $n^{\circ} 7:$ Détail du motif sur P2, du motif ondé sur la baguette moulurée $n^{\circ} 2$, positionnement des clous, vue depuis le nord-ouest)]. Celles-ci reçoivent les solives ou «doublis» disposés en nappes légèrement rayonnantes afin de reprendre l'équerrage des murs.

Les solives sont espacées entre elles d'une distance allant de $25 \mathrm{~cm}$ à $30 \mathrm{~cm}$ environ, ce qui paraît peu par rapport à l'usage en vigueur qui correspondrait plutôt à $40 \mathrm{~cm}$ ou $50 \mathrm{~cm}$ [(Sournia, Bernard ; Vaysettes, Jean-Louis. 1991.); Sournia, Vayssettes, 1991 : 144]. Pour le reste, et suivant ces deux auteurs, le plafond du 9 rue de la Petite-Loge semble bien être conforme à la tradition constructive de tels ouvrages. Les planches ou ais formant le plafond reçoivent un couvre-joint ou listel par en dessous et les intervalles entre les solives sont fermés par des «boujats » ou entrevous. Ceux-ci sont posés légèrement inclinés «[...] afin de permettre une meilleure vision des décors peints qui les recouvrent. » (Sournia, Vayssettes, 1991 : 144) [ (Fig. $n^{\circ} 7$ : Détail du motif sur P2, du motif ondé sur la baguette moulurée $\mathrm{n}^{\circ} 2$, positionnement des clous, vue depuis le nord-ouest) : coupe BB, et (Fig. $\mathrm{n}^{\circ} 1$ : Plan de situation de Montpellier et ses environ vers 17311) : $\left.\mathrm{n}^{\circ} 14\right]$. D'après les auteurs de l'ouvrage, Montpellier, la demeure médiévale, un tel type de plafond est connu localement dès la fin du XIII ${ }^{\mathrm{e}} \mathrm{s}$. ou le début du XIV ${ }^{\mathrm{e}} \mathrm{s}$. et est nommé « listellat et boujettat ».

Dans notre cas, une baguette moulurée fait office de raccord entre les entrevous et les poutres ou les murs. Elles sont au nombre de cinq [ (Fig. ${ }^{\circ} 4$ : Plan du plafond, liste et numérotation des poutres et solives) et (Fig. $\mathrm{n}^{\circ} 7$ : Détail du motif sur P2, du motif ondé sur la baguette moulurée $n^{\circ} 2$, positionnement des clous, vue depuis le nord-ouest)], la moulure $n^{\circ} 4$ étant presque inexistante aujourd'hui. Elles sont fixées aux poutres par l'intermédiaire de clous à tête ronde disposés alternativement sous chaque solive et entre deux solives, leur écartement varient de $11 \mathrm{~cm}$ à $20 \mathrm{~cm}$ (plus précisément $17 \mathrm{~cm}, 11 \mathrm{~cm}$, $20 \mathrm{~cm}, 18 \mathrm{~cm}, 20 \mathrm{~cm}$ et $15 \mathrm{~cm}$ ). Ces moulures présentent un profil semblable pour la modénature générale même si, dans le détail, des différences apparaissent (Fig. $\mathrm{n}^{\circ} 1$ : Plan de situation de Montpellier et ses environ vers 17311) : la moulure se compose donc, de haut en bas :

- d'un quart-de-rond, d'un filet vertical,

- d'une gorge à profil segmentaire,

- d'un filet horizontal,

- d'un quart-de-rond.

Les clous situés sous les solives sont positionnés sur le filet vertical, sous le quart-de-rond haut. Les clous logés entre les solives se nichent au milieu de la gorge centrale (Fig. n 8 : Plan du plafond, repérage des sections sur solives et moulures).

Nous avons déjà signalé la présence d'un escalier sur trompe, placé dans l'angle ouest de la pièce: le raccord entre ces deux structures (escalier et plafond) a fait l'objet d'observations de détail. Le plafond ne semble pas avoir subi de reprises lors de la création de l'escalier, mais au contraire en reprendre les lignes de force dans sa structure. En effet, au niveau des solives S45, S46, S47, on constate que les entrevous suivent la courbe formée par l'escalier. Ce qui donne à penser que le plafond lui est contemporain ou postérieur. 


\section{Les solives}

8 Une attention plus particulière a été portée aux solives constitutives du plafond. Disposées en nappes légèrement rayonnantes pour reprendre l'équerrage des murs de la pièce, elles supportent elles aussi une mouluration en partie basse (Fig. $\mathrm{n}^{\circ} 1$ : Plan de situation de Montpellier et ses environ vers 17310). Le motif mouluré se compose, du haut vers le bas :

- d'un filet horizontal,

- d'une baguette,

- d'un filet vertical, se trouvant de part et d'autre d'une bande en sous-face des solives.

9 Là encore des divergences de détail apparaissent dans la facture des moulures, non significatives cependant, mais simplement représentatives d'un travail manuel. Au niveau de leur dimensionnement, la hauteur générale des solives tourne autour des $18 \mathrm{~cm}$, avec des écarts pouvant aller de $0,2 \mathrm{~cm}$ à $1 \mathrm{~cm}$. Certaines solives sont parfois moins hautes (par exemple, S21 ne fait que $14 \mathrm{~cm}$ de haut); elles reçoivent alors une cale en partie haute destinée à combler cette différence, moyen pour le moins rudimentaire de résoudre le problème... Pour les largeurs, deux modules types semblent se dégager : un module mince allant de $7 \mathrm{~cm}$ à $8 \mathrm{~cm}$ de large, un module large approchant les $10 \mathrm{~cm}$.

10 Nous avons cherché à déterminer si ces deux modules, dans leur répartition ou autrement, avaient une signification dans la structure du plancher. Cela ne paraît pas être le cas sauf peut-être dans un domaine particulier : celui des solives de récupération (Fig. $\mathrm{n}$ ${ }^{\circ} 1$ : Plan de situation de Montpellier et ses environ vers 17312). Il est, en effet, apparu que certaines solives portaient la trace d'un usage antérieur à leur présence dans le plafond du 9 rue de la Petite Loge, pour fermer le vide situé entre les solives, des entrevous sont disposés suivant une légère inclinaison. Ils sont engagés dans une rainure pratiquée dans les solives. Certaines d'entre elles montrent des rainures plus anciennes, visibles au niveau de leur jonction avec les murs et les poutres (Annexes 1 : liste des solives); il y a deux types de rainures observées sur certaines solives: celles correspondant aux entrevous actuels et d'autres, placées à des distances variables par rapport aux précédentes $(15 \mathrm{~cm}, 20 \mathrm{~cm}, 18 \mathrm{~cm}, 8 \mathrm{~cm}, 7 \mathrm{~cm}, 22 \mathrm{~cm}, 14 \mathrm{~cm}, 3 \mathrm{~cm}$...) pouvant être l'indice d'une réfection ou d'un réemploi (Fig. $\mathrm{n}^{\circ} 1$ : Plan de situation de Montpellier et ses environ vers 17313). Il arrive parfois que des entrevous actuels soient positionnés dans des rainures anciennes (S40 et S38) qui s'accompagnent en partie haute d'une encoche devant recevoir le premier listel. [ (Fig. $\mathrm{n}^{\circ} 7$ : Détail du motif sur P2, du motif ondé sur la baguette moulurée $\mathrm{n}^{\circ} 2$, positionnement des clous, vue depuis le nord-ouest), coupe BB ; (Fig. $\mathrm{n}^{\circ} 1$ : Plan de situation de Montpellier et ses environ vers 17313) au premier plan)]. Disposition que l'on ne retrouve pas dans le plancher actuel où il n'y a pas de listel à la jonction entrevous - ais (Fig. $n^{\circ} 8$ : Plan du plafond, repérage des sections sur solives et moulures). Ce type d'observation nous a permis de déterminer le nombre probable des solives de récupération : trente-huit solives sur soixante-trois dont on est sûr qu'elles le soient, ce qui n'interdit pas que d'autres ne montrant pas de traces de rainures anciennes ne puissent également provenir d'une structure antérieurement détruite.

11 Il semblerait, par ailleurs, qu'il y ait une plus forte proportion de solives de récupération dans les modules plus épais. Les solives de $7 \mathrm{~cm}$ à $8 \mathrm{~cm}$ d'épaisseur ne montraient le plus souvent aucune trace d'utilisation plus ancienne. Nous n'avons cependant pas pu dégager de constante à ce niveau, tout au plus une tendance générale. Il n'a pas été possible de 
mettre en relation les variantes perçues au niveau du motif mouluré en partie basse des solives avec une éventuelle récupération de certaines d'entre elles. On ne doit pas perdre de vue que cette décoration moulurée a vraisemblablement dû être réalisée globalement lors de la mise en œuvre du plancher du 9 rue de la Petite-Loge et donc être appliquée aux pièces récupérées antérieurement.

Les solives sont-elles filantes d'une travée à l'autre ou non? Un repérage direct a été effectué sur les solives $\mathrm{S} 42$ et $\mathrm{S} 63$ à travers un entrevous cassé où a été perçu une seule solive filante sur les travées 2 et 3 (Fig. $n^{\circ} 1$ : Plan de situation de Montpellier et ses environ vers 17312). Cette même étude nous montre, en revanche, que les solives S43 et $\mathrm{S} 64$ ne sont pas filantes sur ces deux mêmes travées. Là non plus, il n'y a donc pas de systématisation dans le mode de construction du plancher. Nous avons alors cherché à déterminer quelles solives pouvaient être filantes ou non, à l'aide de plusieurs critères :

- leur positionnement respectif de part et d'autre des poutres P1 et P2,

- leurs dimensions,

- leur état de récupération ou non.

Les résultats, alors obtenus, donnent une série de solives qui seraient filantes du point de vue de la logique constructive, mais pour lesquelles aucun examen direct n'a été réalisé, les entrevous n'étaient, fort heureusement, pas toujours endommagés et ne permettaient donc pas d'avoir une vision directe du phénomène. Il s'avère que seules les travées 3 et 2 auraient reçu des solives filantes, la travée 1 montre un changement d'orientation des solives quasi systématique afin de rattraper l'équerrage des murs. Les dimensions des solives ne correspondent pas non plus entre elles, de part et d'autre de la poutre P1. Ainsi, nous estimons à douze le nombre des solives filantes sur les travées 2 et 3 . Une observation complémentaire a été faite sur les solives que nous pensons être filantes et qui sont aussi de récupération. Les traces d'entrevous anciens, qui peuvent être vus de part et d'autre de la poutre $\mathrm{P} 2$ sur une même solive filante de récupération, montrent révèlent un écartement allant de $46 \mathrm{~cm}$ à $50 \mathrm{~cm}[47 \mathrm{~cm}, 49 \mathrm{~cm}, 46 \mathrm{~cm}, 48 \mathrm{~cm}, 49 \mathrm{~cm}, 50 \mathrm{~cm}$. Annexe 1 : liste des solives et (Fig. $n^{\circ} 1$ : Plan de situation de Montpellier et ses environ vers 17312)]. Ce qui nous laisse envisager que ces solives proviennent d'un même plafond, plus ancien que celui faisant l'objet de la présente étude, supporté par des poutres plus larges que celles que nous avons aujourd'hui.

Par ailleurs, les lignes d'entrevous anciennes ne sont pas parallèles aux lignes entrevous actuelles, et elles-mêmes non parallèles entre elles. Les inclinaisons, tant pour les entrevous anciens que pour ceux présents aujourd'hui, ne semblent pas être toujours identiques.

\section{L'état de conservation}

Ce plancher a bien entendu vécu et souffert. Au niveau structurel, les deux poutres P1 et $\mathrm{P} 2$ ont subi de fortes flèches dans la partie centrale de la pièce, au niveau de l'actuelle cloison encore présente sur place [ (Fig. $\mathrm{n}^{\circ} 7$ : Détail du motif sur P2, du motif ondé sur la baguette moulurée $\mathrm{n}^{\circ} 2$, positionnement des clous, vue depuis le nord-ouest), coupe AA]. De même, les solives ont joué et ne sont plus parfaitement disposées à l'horizontale [ (Fig. $\mathrm{n}^{\circ} 7$ : Détail du motif sur P2, du motif ondé sur la baguette moulurée $\mathrm{n}^{\circ} 2$, positionnement des clous, vue depuis le nord-ouest), coupe BB]. Par ailleurs, des pièces de bois récentes sont venues en renfort ou en remplacement, par exemple, S44 est remplacée par une 
panne moderne, une autre est placée en renfort contre le mur de façade sur la rue de la Petite Loge et supporte les solives.

En outre, des modifications dans l'agencement des lieux sont décelables. Ainsi, dans la travée 3, peut-on remarquer le long du mur une bande de trois ais de large ayant reçu une peinture au plomb grise, postérieurement au décor initial (Fig. $n^{\circ} 1$ : Plan de situation de Montpellier et ses environ vers 17314). On note la présence le long de cette bande, entre les ais 2-41 à 40-60, d'une trace d'humidité qui reprend le tracé d'une cloison plus récente, de $6 \mathrm{~cm}$ de large, encore lisible sur le plafond (Fig. $\mathrm{n}^{\circ} 1$ : Plan de situation de Montpellier et ses environ vers 17315) et ménage contre le mur un couloir de $130 \mathrm{~cm}$ de large, correspondant à la zone peinte à la peinture au plomb grise.

Les ais et les entrevous ont subi des dégradations multiples : certains ont brûlé, d'autres sont noircis, cassés, fendus, ont subi des dégâts des eaux. Le bois, enfin, est parfois vermoulu. Des parties saines au niveau du bois peuvent avoir leur décor effacé. Pour réaliser la figure 16 (Fig. $\mathrm{n}^{\circ} 1$ : Plan de situation de Montpellier et ses environ vers 17316), nous avons procédé par zonage et avons donc dû regrouper certains paramètres d'usure ou de dégradation. Nous avons ainsi isolé les endroits ayant subi le feu et ceux étant vermoulus. Mais nous avons regroupé sous une seule famille ceux qui sont abîmés à des degrés divers: noircis, cassés, fendus, dégât des eaux. Les éléments noircis sont éventuellement significatifs de l'usage qui fut fait de la pièce: zones d'éclairage à la bougie, par exemple. Notons donc que deux séries de huit ais noircis consécutifs sont présentes en partie médiane des travées 2 et 3 , à proximité du mur nord-est de la pièce. L'annexe 2 (Liste des ais) donne le détail de ces états.

18 Nous signalons que la panne moderne posée en renfort contre le mur de façade sur la rue de la Petite-Loge est elle-même partiellement brûlée. Les actions du feu peuvent donc être relativement récentes.

\section{Le décor}

19 Ce plancher a reçu, outre les moulurations, un décor peint. Celui-ci est disposé suivant des emplacements spécifiques. Bien que les motifs soient dans un registre non figuratif, à modénature aléatoire, on peut néanmoins parler de programme décoratif.

\section{Les ais}

Les ais supportent un décor de motifs non figuratifs, semblant vouloir représenter le veinage du bois [ (Fig. $n^{\circ} 2$ : Vue générale sur la deuxième travée, de S27 à S32, vue depuis le nord-ouest), (Fig. $\mathrm{n}^{\circ} 1:$ Plan de situation de Montpellier et ses environ vers 17315), (Fig. $\mathrm{n}^{\circ} 1$ : Plan de situation de Montpellier et ses environ vers 17317), (Fig. $\mathrm{n}^{\circ} 1$ : Plan de situation de Montpellier et ses environ vers 17318), (Fig. $n^{\circ} 1$ : Plan de situation de Montpellier et ses environ vers 17319) et (Fig. $n^{\circ} 2$ : Vue générale sur la deuxième travée, de S27 à S32, vue depuis le nord-ouesto)]. Il s'avèrerait, d'après Jean-Louis Vayssettes, qu'il s'agisse là d'un décor exécuté au chiffon. Un badigeon plus clair a d'abord été apposé, semble-t-il, après le montage du plancher. En effet, les angles et les bords des ais $n$ 'ont pas subi cette application, comme si listels et solives venaient entraver le geste du décorateur [ (Fig. $\mathrm{n}^{\circ} 8$ : Plan du plafond, repérage des sections sur solives et moulures), (Fig. $n^{\circ} 1$ : Plan de situation de Montpellier et ses environ vers 17315), (Fig. $n^{\circ} 1$ : Plan de 
situation de Montpellier et ses environ vers 17319), (Fig. $\mathrm{n}^{\circ} 2$ : Vue générale sur la deuxième travée, de S27 à S32, vue depuis le nord-ouest0)]. Par ailleurs, les anciennes rainures destinées au maintien des entrevous n'ont pas été masquées par une couche de fond coloré, le bois est resté brut à ces endroits.

Les listels venant faire couvre-joint entre les ais ont reçu une bande peinte en sous-face uniquement (Fig. $n^{\circ} 1:$ Plan de situation de Montpellier et ses environ vers 17319).

\section{Les entrevous}

22 Ils ont été couvert par le même type de décor que les ais [ (Fig. n ${ }^{\circ} 8$ : Plan du plafond, repérage des sections sur solives et moulures), (Fig. $\mathrm{n}^{\circ} 1$ : Plan de situation de Montpellier et ses environ vers 17319), (Fig. n² : Vue générale sur la deuxième travée, de S27 à S32, vue depuis le nord-ouest1), (Fig. $\mathrm{n}^{\circ} 2$ : Vue générale sur la deuxième travée, de $\mathrm{S} 27$ à $\mathrm{S} 32$, vue depuis le nord-ouest2), (Fig. $\mathrm{n}^{\circ} 2$ : Vue générale sur la deuxième travée, de S27 à S32, vue depuis le nord-ouest3)]. Comme pour les ais, on peut se demander s'il s'agit là de la couleur d'origine ou si celle-ci a subi des altérations. Visible sur les figures 19, 21 et 22, la jonction, au niveau des poutres, entre les ais et les entrevous est traitée au moyen d'une bande peinte formant listel.

\section{Les baguettes moulurées et les poutres}

Ici, le décor utilise la modénature des moulures, les quarts-de-rond reçoivent une bande peinte unie, la gorge centrale est le lieu d'une décoration de motifs ondés [ (Fig. $\mathrm{n}^{\circ} 8$ : Plan du plafond, repérage des sections sur solives et moulures), (Fig. $\mathrm{n}^{\circ} 1$ : Plan de situation de Montpellier et ses environ vers 17313), (Fig. $\mathrm{n}^{\circ} 1$ : Plan de situation de Montpellier et ses environ vers 17319), (Fig. $\mathrm{n}^{\circ} 2$ : Vue générale sur la deuxième travée, de S27 à S32, vue depuis le nord-ouest1), (Fig. $\mathrm{n}^{\circ} 2$ : Vue générale sur la deuxième travée, de S27 à S32, vue depuis le nord-ouest3)].

On a observé sur la poutre P2 un décor qui devait être de même facture que celui des ais et des entrevous, visible partiellement en partie haute. La partie basse prend sur une moulure plate une bande peinte formant listel [ (Fig. n ${ }^{\circ} 8$ : Plan du plafond, repérage des sections sur solives et moulures), (Fig. $n^{\circ} 2$ : Vue générale sur la deuxième travée, de S27 à S32, vue depuis le nord-ouest3)].

\section{Les solives}

Les joues des solives reçoivent un motif assez semblable à celui des ais et des entrevous (Fig. ${ }^{\circ} 1$ : Plan de situation de Montpellier et ses environ vers 17313). Sur leur sous-face se déroule un motif ondé [ (Fig. ${ }^{\circ} 1$ : Plan de situation de Montpellier et ses environ vers 17317), (Fig. $n^{\circ} 1:$ Plan de situation de Montpellier et ses environ vers 17318), (Fig. $n^{\circ}$ 1 : Plan de situation de Montpellier et ses environ vers 17319), (Fig. $n^{\circ} 2:$ Vue générale sur la deuxième travée, de S27 à S32, vue depuis le nord-ouest1), (Fig. $\mathrm{n}^{\circ} 2$ : Vue générale sur la deuxième travée, de S27 à S32, vue depuis le nord-ouest2)]. Les baguettes sont uniformément peintes d'une bande sombre. À la jonction solives-baguettes moulurées, la sous-face des solives reçoit une bande peinte formant listel [ (Fig. ${ }^{\circ} 1$ : Plan de situation de Montpellier et ses environ vers 17319), (Fig. $\mathrm{n}^{\circ} 2$ : Vue générale sur la deuxième travée, 
de $\mathrm{S} 27$ à S32, vue depuis le nord-ouest1), (Fig. $n^{\circ} 2$ : Vue générale sur la deuxième travée, de S27 à S32, vue depuis le nord-ouest2)]. du début deuxième quart du XVIII $s$.

\section{Le mur} Louis. 1991.); Sournia, Vayssettes, 1991 : 174].

\section{Conclusion} informations techniques et historiques. vers la fin du XVIII ${ }^{\mathrm{e}}$ s. au moins. 1980.), (Peyron, Jacques. 1990.)]

En ce qui concerne la mouluration des solives, un exemple similaire est daté du XVIII ${ }^{\mathrm{e}} \mathrm{s}$. à Pont du Château (Puy de Dôme), [(Peyron, Jacques. 1990.); Peyron, 1990 : 179, fig. 43], sans que l'on puisse prétendre étendre cette datation à l'exemple qui nous intéresse ici.

À titre anecdotique, signalons plusieurs armoires en bois peintes, vues au musée alsacien de Strasbourg. Leurs motifs sont bien sûr différents de ceux étudiés ici, mais tendent également à reproduire le veinage du bois. D'après le conservateur de ce musée, ces décors seraient réalisés avec du sang de bœuf frais délayé dans du vinaigre. Ce mélange devient brun foncé en séchant. Les outils utilisés seraient des rondelles de pomme de terre (pour faire des ronds parfaits), le chiffon, le pinceau... Ces armoires seraient datées

8 Étaient encore perceptibles, en partie haute du mur ménageant accès à la pièce, une bande sombre peinte formant listel, ainsi que des motifs ondés (Fig. $n^{\circ} 2$ : Vue générale sur la deuxième travée, de S27 à S32, vue depuis le nord-ouest4). Il semblerait donc que le programme décoratif se soit poursuivi au moins en partie haute des murs, la possibilité d'ateliers réalisant plafonds et murs dans un même programme iconographique est signalée dans Montpellier, la demeure médiévale [(Sournia, Bernard ; Vaysettes, Jean-

L'étude a permis de caractériser assez précisément cet ouvrage (pièces utilisées, structure, décoration et stylistique, remplois, etc.) et de sauvegarder l'essentiel des

Ce plafond, malgré un aspect «bricolage » par endroits (la présence de cales non peintes pour rattraper les différences de hauteurs de certaines solives par exemple) et la forte probabilité d'un important remploi de solives ( $60 \%$ environ), montre une grande unité tant dans la structure elle-même que dans la décoration. Nous n'avons, cependant, pas pu trouver de motifs semblables ailleurs, du moins dans le domaine du bâti, ce qui nous interdit toute possibilité de datation par le biais de la stylistique. Seule l'histoire précise de l'architecture (date de création de l'escalier sur trompe et de rehaussement du plafond) pourrait donner une indication chronologique, la structure même du plancher ayant eu une vie longue dans la région : signalée dès la fin $\mathrm{du} \mathrm{XIII}^{\mathrm{e}}$ s., elle perdure jusque

31 Cet exemple d'étude prendra tout son sens lorsque nous disposerons sur Montpellier et sa région de plusieurs autres matériaux ou exemplaires, permettant d'observer de manière fiable - sur la base d'une véritable suite - les aspects techniques et chronologiques.

[(Sournia, Bernard ; Vaysettes, Jean-Louis. 1991.), (Aslet, Gustave ; Jeannin, Jules. 1910.), (Pérouse de Montclos, Jean-Marie. 1972.), (Fréal, Jacques. 1977.), (Lhuisset, Christian. 


\section{Annexe 1 : Liste des solives}

S3 - Travée 1

$8,50 \mathrm{~cm} / 18,10 \mathrm{~cm}$.

Très vermoulue.

De récupération.

S4 - Travée 1

$8,00 \mathrm{~cm} / 17,10 \mathrm{~cm}$.

Très vermoulue.

De récupération.

S5 - Travée 1

$8,60 \mathrm{~cm} / 17,10 \mathrm{~cm}$.

Vermoulue.

De récupération.

S6 - Travée 1

$9,50 \mathrm{~cm} / 17,00 \mathrm{~cm}$.

Très vermoulue.

De récupération.

S7 - Travée 1

$9,00 \mathrm{~cm} / 17,10 \mathrm{~cm}$.

Vermoulue.

De récupération.

S8 - Travée 1

$9,50 \mathrm{~cm} / 17,50 \mathrm{~cm}$.

Très vermoulue + feu.

De récupération.

S9 - Travée 1 - Section $n^{\circ} 9$.

$8,80 \mathrm{~cm} / 18,10 \mathrm{~cm}$.

Rongée par l'humidité contre le mur.

De récupération.

S10 - Travée 1 - Section $n^{\circ} 10$.

$9,50 \mathrm{~cm} / 18,70 \mathrm{~cm}$.

Très vermoulue + appui sur mur détérioré.

De récupération.

S11 - Travée 1

Masquée par le faux-plafond actuel.

Vermoulue.

De récupération. 
S12 - Travée 1

Noyée dans la cloison.

Vermoulue.

S13 - Travée 1

$7,00 \mathrm{~cm} / 16,00 \mathrm{~cm}$.

Vermoulue.

S14 - Travée 1

$8,20 \mathrm{~cm} / 16,70 \mathrm{~cm}$.

Très vermoulue en partie haute.

De récupération.

S15 - Travée 1

$7,50 \mathrm{~cm} / 17,50 \mathrm{~cm}$.

État sanitaire bon, mais poutre noircie.

S16 - Travée 1

$8,00 \mathrm{~cm} / 18,00 \mathrm{~cm}$.

Bon état sanitaire.

De récupération.

S17 - Travée 1

$7,50 \mathrm{~cm} / 17,70 \mathrm{~cm}$.

Vermoulue en partie haute.

S18 - Travée 1

$7,50 \mathrm{~cm} / 18,00 \mathrm{~cm}$.

Bon état sanitaire même si sous-face abîmée.

S19 - Travée 1

$7,00 \mathrm{~cm} / 17,20 \mathrm{~cm}$.

Vermoulue + noircie

S20 - Travée 1 - Section $n^{\circ} 11$.

$7,80 \mathrm{~cm} / 17,5 \mathrm{~cm}$.

Très vermoulue + noircie.

De récupération.

S21 - Travée 1 - Section $n^{\circ} 12$.

$7,40 \mathrm{~cm} / 16,20 \mathrm{~cm}$.

Vermoulue + noircie + très abîmée au niveau de l'ancrage dans le mur.

S22 - Travée 1

$8,50 \mathrm{~cm} / 18,40 \mathrm{~cm}$.

Très vermoulue + noircie + sous-face très abîmée (mouluration presque totalement disparue).

De récupération. 
S23 - Travée 1

$6,50 \mathrm{~cm} / 17,20 \mathrm{~cm}$.

Abîmée en sous-face + noircie.

Ne repose plus que sur un coin au niveau de l'ancrage dans le mur, la panne moderne ayant brûlée.

S24 - Travée 2

$7,50 \mathrm{~cm} / 17,60 \mathrm{~cm}$

Vermoulue + feu.

De récupération.

S25 - Travée 2

$8,50 \mathrm{~cm} / 17,10 \mathrm{~cm}$.

Vermoulue + feu.

De récupération.

Pourrait être filante avec S46.

S26 - Travée 2

$9,00 \mathrm{~cm} / 17,00 \mathrm{~cm}$.

Vermoulue.

De récupération.

S27 - Travée 2

$7,00 \mathrm{~cm} / 17,60 \mathrm{~cm}$.

Bon état sanitaire.

Pourrait être filante avec S48.

S28 - Travée 2

$9,00 \mathrm{~cm} / 18,00 \mathrm{~cm}$.

Vermoulue.

De récupération.

Pourrait être filante avec S49.

S29 - Travée 2

$9,50 \mathrm{~cm} / 18,00 \mathrm{~cm}$.

Vermoulue + feu + fendue

De récupération.

S30 - Travée 2 - Section $n^{\circ} 7$.

$9,40 \mathrm{~cm} / 17,50 \mathrm{~cm}$.

Vermoulue par endroit + motifs abîmés.

De récupération.

S31 - Travée 2 - Section $n^{\circ} 8$.

$7,00 \mathrm{~cm} / 18,00 \mathrm{~cm}$.

État sanitaire bon. 
De récupération.

S32 - Travée 2

$8,00 \mathrm{~cm} / 17,50 \mathrm{~cm}$.

Très vermoulue.

De récupération.

Pourrait être filante avec S53.

S33 - Travée 2

Noyée dans la cloison.

De récupération.

S34 - Travée 2

$6,50 \mathrm{~cm} / 17,00 \mathrm{~cm}$.

Vermoulue + motifs abîmés.

Pourrait être filante avec S54.

S35 - Travée 2

$8,00 \mathrm{~cm} / 17,10 \mathrm{~cm}$.

Très vermoulue + cassée dans le sens de la flèche.

De récupération.

Pourrait être filante avec S56.

S36 - Travée 2

$7,30 \mathrm{~cm} / 17,00 \mathrm{~cm}$.

Très vermoulue + cassée dans le sens de la flèche.

Pourrait être filante avec S57.

S37 - Travée 2

$6,50 \mathrm{~cm} / 17,10 \mathrm{~cm}$

État sanitaire bon.

Pourrait être filante avec S58.

S38 - Travée 2

$9,00 \mathrm{~cm} / 17,50 \mathrm{~cm}$.

Très vermoulue.

De récupération.

Pourrait être filante avec S59.

Écart entre les anciennes lignes d'entrevous de part et d'autre de P2 :49,00 cm.

S39 - Travée 2

$10,00 \mathrm{~cm} / 18,00 \mathrm{~cm}$.

Très vermoulue.

De récupération.

Pourrait être filante avec S60.

S40 - Travée 2 
$8,40 \mathrm{~cm} / 18,00 \mathrm{~cm}$.

Bon état sanitaire.

De récupération.

Pourrait être filante avec S61.

Écart entre les anciennes lignes d'entrevous de part et d'autre de P2:50cm.

S41 - Travée 2

$8,00 \mathrm{~cm} / 16,70 \mathrm{~cm}$.

Vermoulue.

De récupération.

S42 - Travée 2

$6,00 \mathrm{~cm} / 17,00 \mathrm{~cm}$

Commence à être vermoulue.

Filante avec S63 : observation faite à travers un entrevous cassé.

S43 - Travée 2

$7,50 \mathrm{~cm} / 16,70 \mathrm{~cm}$.

Vermoulue + brûlée.De récupération.

Non filante : observation faite à travers un entrevous cassé.

S44 - Travée 2

Est remplacée par une panne moderne.

S45 - Travée 3

$9,50 \mathrm{~cm} / 18,00 \mathrm{~cm}$

Vermoulue en partie haute.

Peinture au plomb contre le mur.

S46 - Travée 3

$8,50 \mathrm{~cm} / 17,50 \mathrm{~cm}$.

Vermoulue en partie haute.Peinture au plomb contre le mur.

De récupération.

Pourrait être filante avec S25.

Écart entre les anciennes lignes d'entrevous de part et d'autre de $\mathrm{P} 2: 50,00 \mathrm{~cm}$.

S47 - Travée 3 - Section $n^{\circ} 1$.

$9,00 \mathrm{~cm} / 17,80 \mathrm{~cm}$.

État sanitaire bon.Peinture au plomb contre le mur.

De récupération.

S48 - Travée 3 - Section $n^{\circ} 2$.

$7,00 \mathrm{~cm} / 17,80 \mathrm{~cm}$.

État sanitaire bon.

Pourrait être filante avec S27.

S49 - Travée 3 - Section $n^{\circ} 3$. 
$9,10 \mathrm{~cm} / 18,00 \mathrm{~cm}$.

État sanitaire bon.

Peinture au plomb contre le mur.

De récupération.

Pourrait être filante avec S28.

Écart entre les anciennes lignes d'entrevous de part et d'autre de P2:49,00cm.

S50 - Travée 3 - Section $n^{\circ} 4$.

$7,30 \mathrm{~cm} / 17,00 \mathrm{~cm}$.

Feu.

Peinture au plomb contre le mur.

S51 - Travée 3

$8,60 \mathrm{~cm} / 17,00 \mathrm{~cm}$.

Bon état sanitaire.

Peinture au plomb contre le mur.

De récupération.

S52 - Travée 3 - Section $n^{\circ} 5$.

$7,20 \mathrm{~cm} / 18,00 \mathrm{~cm}$.

Feu.

Peinture au plomb contre le mur.

S53 - Travée 3

$8,00 \mathrm{~cm} / 18,70 \mathrm{~cm}$.

Humidité.

Peinture au plomb contre le mur.

De récupération.

Pourrait être filante avec S32.

Écart entre les anciennes lignes d'entrevous de part et d'autre de P2 : 48,00 cm.

S54 - Travée 3

$7,00 \mathrm{~cm} / 17,70 \mathrm{~cm}$.

Humidité + fendue.

Peinture au plomb contre le mur.

S55 - Travée 3

$6,50 \mathrm{~cm} / 17,10 \mathrm{~cm}$.

État sanitaire bon malgré une trace d'humidité contre la cloison supposée.

S56 - Travée 3

$7,40 \mathrm{~cm} / 16,90 \mathrm{~cm}$.

État sanitaire bon malgré une trace d'humidité contre la cloisonsupposée.

Pourrait être filante avec S35.

S57 - Travée 3 
$7,50 \mathrm{~cm} / 16,30 \mathrm{~cm}$.

Feu + refort bois moderne contre le mur.

Pourrait être filante avec S36.

S58 - Travée 3

$6,50 \mathrm{~cm} / 17,50 \mathrm{~cm}$

Brûlée contre le mur.

Pourrait être filante avec S37.

S59 - Travée 3

$9,20 \mathrm{~cm} / 17,50 \mathrm{~cm}$

Très vermoulue.

De récupération.

Pourrait être filante avec S38.

Écart entre les anciennes lignes d'entrevous de part et d'autre de P2 : 46,00 cm.

S60 - Travée 3 - Section $n^{\circ} 6$.

$10,00 \mathrm{~cm} / 17,90 \mathrm{~cm}$.

Vermoulue + feu.

De récupération.

Pourrait être filante avec S39.

Écart maximum possible entre les anciennes lignes d'entrevous de part et d'autre de P2: $49,00 \mathrm{~cm}$.

S61 - Travée 3

$8,80 \mathrm{~cm} / 18,50 \mathrm{~cm}$.

Feu.

De récupération.

Pourrait être filante avec S40.

Écart entre les anciennes lignes d'entrevous de part et d'autre de P2: 47,00 cm.

S62 - Travée 3

$8,20 \mathrm{~cm} / 16,70 \mathrm{~cm}$.

Vermoulue + brûlée.

S63 - Travée 3

$6,00 \mathrm{~cm} / 17,00 \mathrm{~cm}$.

Vermoulue + feu.

Filante avec $\$ 42$ : observation faite à travers un entrevous cassé.

S64 - Travée 3

$10,00 \mathrm{~cm} / 17,70 \mathrm{~cm}$.

Brûlée.

S'ancre de 20,00 cm dans le mur, dans son deuxième parement.

De récupération. 


\section{Annexe 2 : Liste des ais}

S65 - Travée 3

Noyée dans le mur.

Très vermoulue.

De récupération.

Non filante : observation faite à travers un entrevous cassé.

Ne s'ancre que dans le premier parement du mur.

$\begin{array}{ll}300 & 001 \text { - Bon } \\ 301 & 002 \text { - Abîmé } \\ 302 & 003 \text { - Bon } \\ 303 & 004 \text { - Abîmé } \\ 304 & 005 \text { - Bon } \\ 305 & 006 \text { - Abîmé } \\ 306 & 007 \text { - Bon } \\ 307 & 008 \text { - Abîmé } \\ 308 & 009 \text { - Bon } \\ 309 & 010 \text { - Noirci } \\ 310 & 011 \text { - Brûlé } \\ 311 & 012 \text { - Brûlé } \\ 312 & 013 \text { - Bon } \\ 313 & 014 \text { - Bon } \\ 314 & 015 \text { - Bon } \\ 315 & 016 \text { - Bon } \\ 316 & 017 \text { - Abîmé } \\ 317 & 018 \text { - Troué019 - Fendu } \\ 318 & 020 \text { - Troué } \\ 319 & 021 \text { - Vermoulu } \\ 320 & 022 \text { - Bon } \\ 321 & 023 \text { - Vermoulu } \\ 322 & 024 \text { - Bon } \\ 323 & 025 \text { - Effacé } \\ 324 & 026 \text { - Bon } \\ 325 & 027 \text { - Effacé } \\ 326 & 028 \text { - Bon } \\ 327 & 029 \text { - Effacé } \\ 328 & 030 \text { - Bon } \\ 30.0\end{array}$




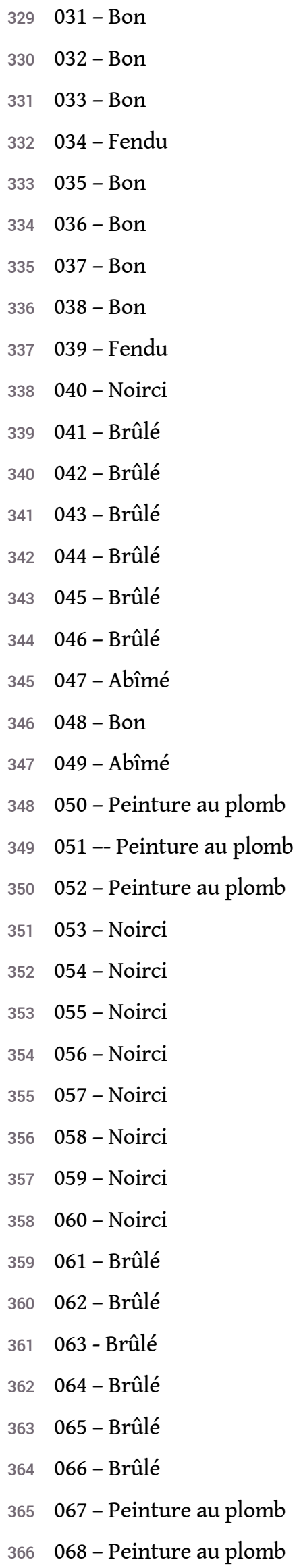




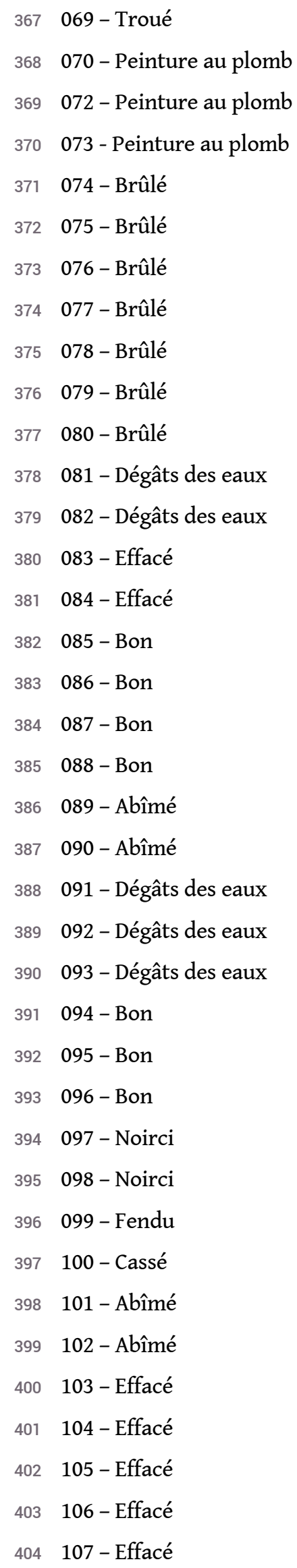




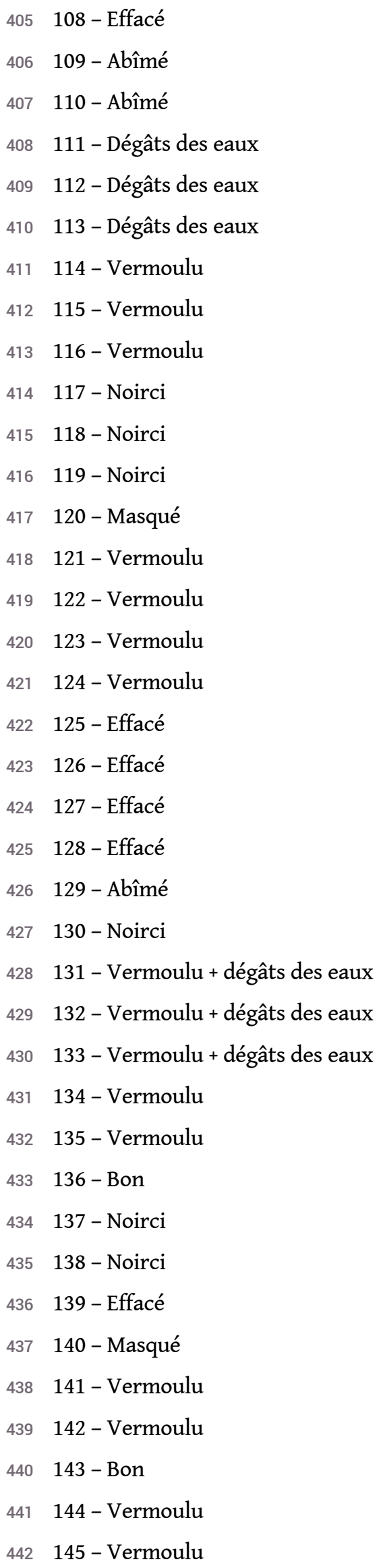




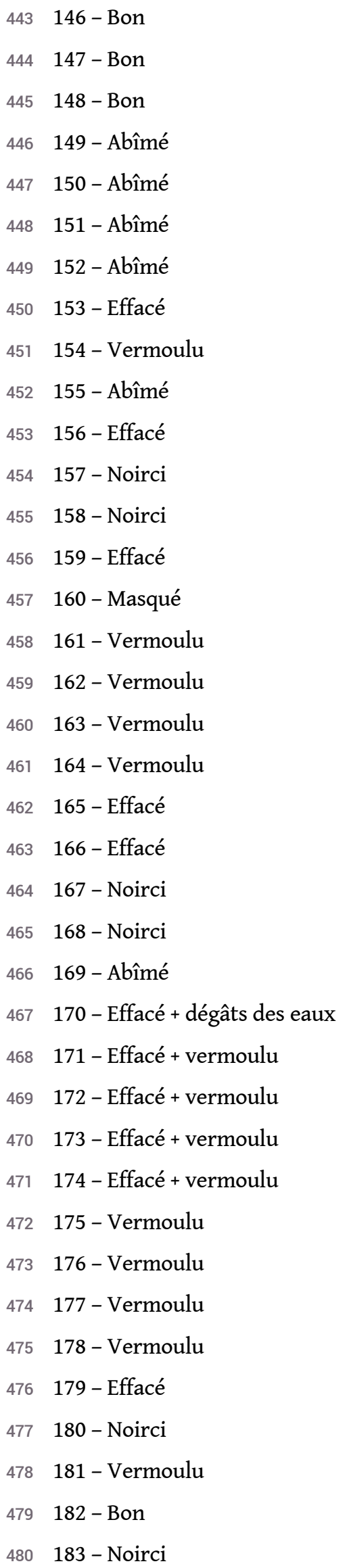




\begin{tabular}{|c|c|}
\hline 481 & 184 - Noirci \\
\hline 482 & 185 - Noirci \\
\hline 483 & $186-$ Bon \\
\hline 484 & $187-$ Bon \\
\hline 485 & $188-$ Bon \\
\hline 486 & 189 - Abîmé \\
\hline 487 & 190 - Noirci \\
\hline 488 & 191 - Noirci \\
\hline 489 & 192 - Abîmé \\
\hline 490 & 193 - Abîmé \\
\hline 491 & $194-$ Bon \\
\hline 492 & $195-$ Bon \\
\hline 493 & 196 - Cassé \\
\hline 494 & 197 - Cassé \\
\hline 495 & 198 - Vermoulu \\
\hline 496 & 199 - Bon \\
\hline 497 & $200-$ Fendu \\
\hline 498 & 201 - Effacé \\
\hline 499 & 202 - Effacé \\
\hline 500 & 203 - Effacé \\
\hline 501 & 204 - Effacé \\
\hline 502 & 205 - Noirci \\
\hline 503 & 206 - Noirci \\
\hline 504 & 207 - Noirci \\
\hline 505 & 208 - Effacé \\
\hline 506 & 209 - Masqué \\
\hline 507 & 210 - Effacé \\
\hline 508 & 211 - Effacé \\
\hline 509 & 212 - Effacé \\
\hline 510 & 213 - Noirci \\
\hline 511 & $214-$ Bon \\
\hline 512 & 215 - Effacé \\
\hline 513 & 216 - Noirci \\
\hline 514 & 217 - Noirci \\
\hline 515 & 218 - Effacé \\
\hline 516 & 219 - Cassé \\
\hline 517 & 220 - Troué \\
\hline 18 & 221 - Abîmé \\
\hline
\end{tabular}



$519222-$ Bon
$520223-$ Bon
$521 \quad 224-$ Fendu
522225 - Fendu
523226 - Effacé
524227 - Effacé
525228 - Effacé
526229 - Masqué
527230 - Abîmé
528231 - Effacé
529232 - Effacé
530233 - Effacé
531234 - Effacé
532235 - Bon
533236 - Effacé
$534 \quad 237$ - Effacé
535238 - Effacé
536239 - Effacé
537240 - Troué
$538241-$ Bon
$539242-$ Bon
$540243-$ Bon
$541244-$ Bon
$542 \quad 245$ - Effacé
543246 - Effacé
$544 \quad 247$ - Noirci
545248 - Effacé
546249 - Masqué
547250 - Effacé
548251 - Effacé
549252 - Effacé
550253 - Effacé
551254 - Effacé
552255 - Effacé
$553256-$ Bon
554257 - Abîmé
555258 - Abîmé
556259 - Cassé 


$$
\begin{aligned}
& 557260 \text { - Cassé } \\
& 558 \quad 261 \text { - Noirci } \\
& 559262 \text { - Vermoulu } \\
& 560263 \text { - Vermoulu } \\
& 561264 \text { - Vermoulu } \\
& 562265 \text { - Effacé } \\
& 563266 \text { - Effacé } \\
& 564267 \text { - Effacé } \\
& 565268 \text { - Effacé } \\
& 566269 \text { - Masqué } \\
& 567270-\text { Bon } \\
& 568271-\text { Bon } \\
& 569272-\text { Bon } \\
& 570273-\text { Bon } \\
& 571274 \text { - Bon } \\
& 572275-\text { Bon } \\
& 573276-\text { Bon } \\
& 574277 \text { - Bon } \\
& 575278 \text { - Abîmé } \\
& 576279 \text { - Abîmé } \\
& 577280 \text { - Fendu } \\
& 578281-\text { Bon } \\
& 579282-\text { Bon } \\
& 580283 \text { - Bon } \\
& 581284-\text { Bon } \\
& 582285-\text { Bon } \\
& 583286-\text { Bon } \\
& 584287-\text { Bon } \\
& 585288 \text { - Effacé } \\
& 586289 \text { - Masqué } \\
& 587290 \text { - Bon } \\
& 588291 \text { - Bon } \\
& 589292-\text { Bon } \\
& 590293-\text { Bon } \\
& 591294-\text { Bon } \\
& 592295-\text { Bon2 } \\
& 593296-\text { Bon } \\
& 594297-\text { Bon }
\end{aligned}
$$




\section{Annexe 3 : Liste des entrevous}

298 - Fendu

299 - Fendu

300 - Fendu

S03/S04 : Cassé

S04/S05 : Abîmé

S05/S06 : Abîmé

S06/S07 : Abîmé

S07/S08 : Abîmé

S08/S09 : Abîmé

S09/S10 : Cassé

S10/S11 : Cassé

S11/S12: Masqué

S12/S13 : Abîmé

S13/S14 : Abîmé

S14/S15 : Abîmé

S15/S16 : Abîmé

S16/S17 : Bon

S17/S18: Bon

S18/S19: Cassé

S19/S20 : Abîmé

S20/S21 : Abîmé

S21/S22: Cassé

Sur travée 1 , contre la poutre P1 : S22/S23 : Cassé

S03/S04 : Abîmé

S04/S05 : Abîmé

S05/S06 : Abîmé

S06/S07 : Bon

S07/S08: Bon

S08/S09: Cassé

S09/S10 : Cassé

S10/S11 : Cassé

S11/S12 : Masqué

S12/S13 : Bon

$\mathrm{S} 13 / \mathrm{S} 14:$ Bon

Sur travée 1, contre le mur de façade sur la rue de la Petite-Loge : 
671

672

704 S46/S47 : Brûlé

705 S47/S48: Brûlé

S31/S32 : Bon

$\mathrm{S} 33 / \mathrm{S} 34$ : Bon

S34/S35 : Bon

S35/S36: Bon

S37/S38: Bon

S40/S41: Bon

S41/S42 : Cassé

45/S46 : Bon

S47/S48: Bon

S50/S51 : Noirci

S51/S52 : Fendu

S52/S53: Bon

S54/S55 : Bon

S55/S56:Bon

S56/S57 : Bon

S57/S58: Bon

S58/S59 : Bon

S59/S60 : Fendu

S60/S61 : Bon

S61/S62 : Bon

S62/S63 : Cassé

S63/S64 : Cassé

S64/S65 : Cassé

S45/S46 : Brûlé
S32/S33 : Masqué

S36/S37 : Abîmé

S38/S39 : Abîmé

S39/S40 : Abîmé

S42/S43 : Abîmé

S43/S44 : Masqué

Sur travée 3, contre poutre P2 :

S46/S47 : Vermoulu

S48/S49 : Vermoulu

S49/S50 : Vermoulu

S53/S54 : Abîmé

Sur travée 3, Contre le mur : 
S48/S49: Masqué

S49/S50 : Masqué

S50/S51: Masqué

S51/S52: Masqué

S52/S53 : Peinture au plomb

S53/S54 : Peinture au plomb

S54/S55 : Peinture au plomb

S55/S56: Cassé

S56/S57 : Brûlé

S57/S58 : Brûlé

S58/S59 : Brûlé

S59/S60 : Brûlé

S60/S61 : Brûlé

S61/S62 : Brûlé

S62/S63 : Brûlé

S63/S64 : Brûlé

S64/S65 : Cassé

\section{BIBLIOGRAPHIE}

Pérouse de Montclos, Jean-Marie. 1972 : « Architecture : méthode et vocabulaire », Inventaire général des monuments et des richesses artistiques de la France, Paris, ministère des Affaires culturelles, Imprimerie nationale, col. Principes d'analyse scientifique, 2 vol, 234 p., 1122 fig.

Sournia, BernardVayssettes, Jean-Louis. 1991 : Montpellier : la demeure médiévale, Paris, Imprimerie nationale, 256 p, coll. Études du patrimoine, 1.

Peyron, Jacques. 1990 : La salle armoiriée moderne du XIVe siècle à la Révolution, Imprimerie Presses Midi-Pyrénées, Col. Couleur et habitation.

Sournia, BernardVayssettes, Jean-Louis. 1991 : Montpellier : la demeure médiévale, Paris, Imprimerie nationale, 256 p, coll. Études du patrimoine, 1.

Sournia, BernardVayssettes, Jean-Louis. 1991 : Montpellier : la demeure médiévale, Paris, Imprimerie nationale, 256 p, coll. Études du patrimoine, 1.

Oslet, GustaveJeannin, Jules. 1910 : « Traité de menuiserie », Encyclopédie théorique et pratique des connaissances civiles et militaires, Partie civile, $5^{\mathrm{e}}$ partie, tome 1, (3 tomes reliés en 2 volumes), Paris, Fanchon et Artus, $556 \mathrm{p}$.

Pérouse de Montclos, Jean-Marie. 1972 : « Architecture : méthode et vocabulaire », Inventaire général des monuments et des richesses artistiques de la France, Paris, ministère des Affaires culturelles, Imprimerie nationale, col. Principes d'analyse scientifique, 2 vol, 234 p., 1122 fig. 
Fréal, Jacques. 1977 : L'architecture paysanne en France, La maison, Ed. Serg, 375 p., 427 fig.

Lhuisset, Christian. 1980 : L'architecture rurale en Languedoc, en Roussillon, Les Provinciades éd., 400 p., 744 fig.

Peyron, Jacques. 1990 : La salle armoiriée moderne du XIV siècle à la Révolution, Imprimerie Presses Midi-Pyrénées, Col. Couleur et habitation.

\section{ANNEXES}

Fig. $\mathrm{n}^{\circ} 1$ : Plan de situation de Montpellier et ses environ vers 1731

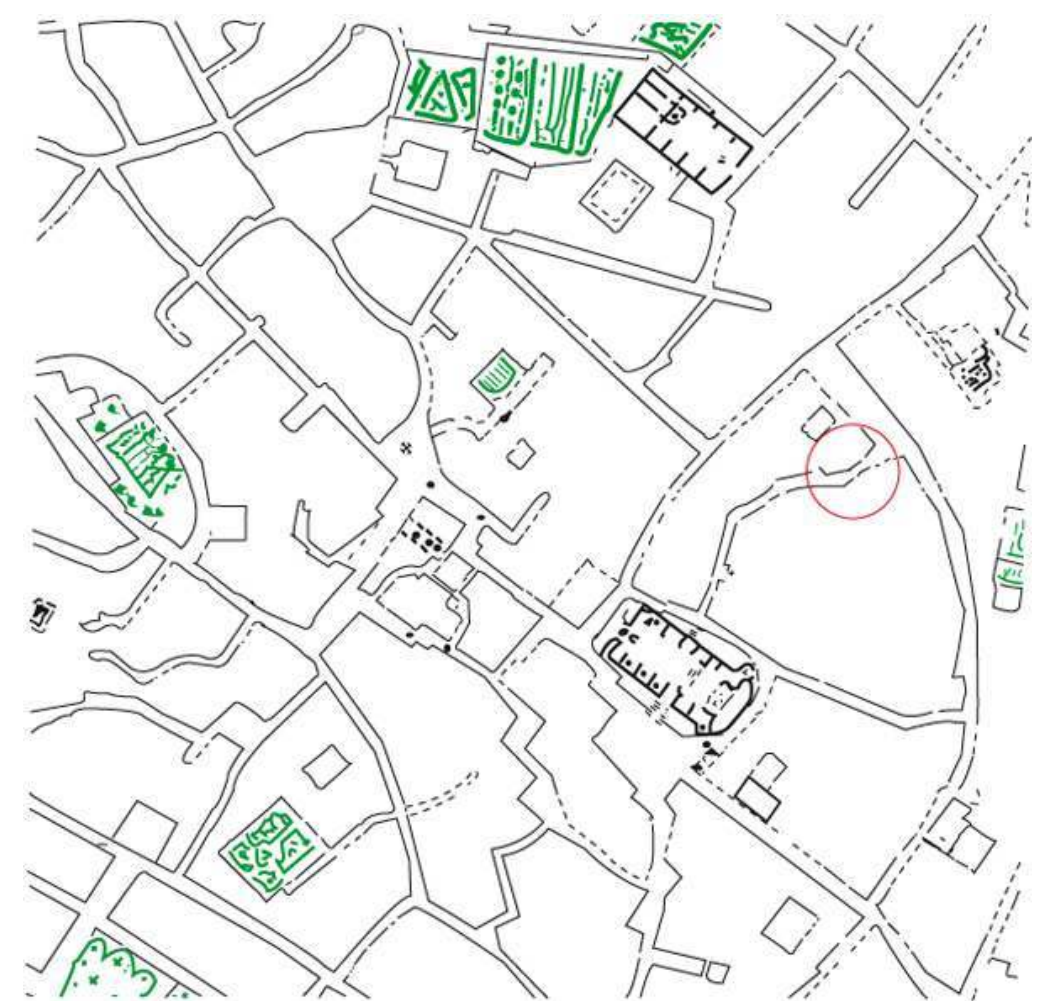

Auteur(s) : Musée languedocien de Montpellier. Crédits : ADLFI - Musée languedocien de Montpellier (2003) 
Fig. $n^{\circ} 2$ : Vue générale sur la deuxième travée, de $S 27$ à $S 32$, vue depuis le nord-ouest

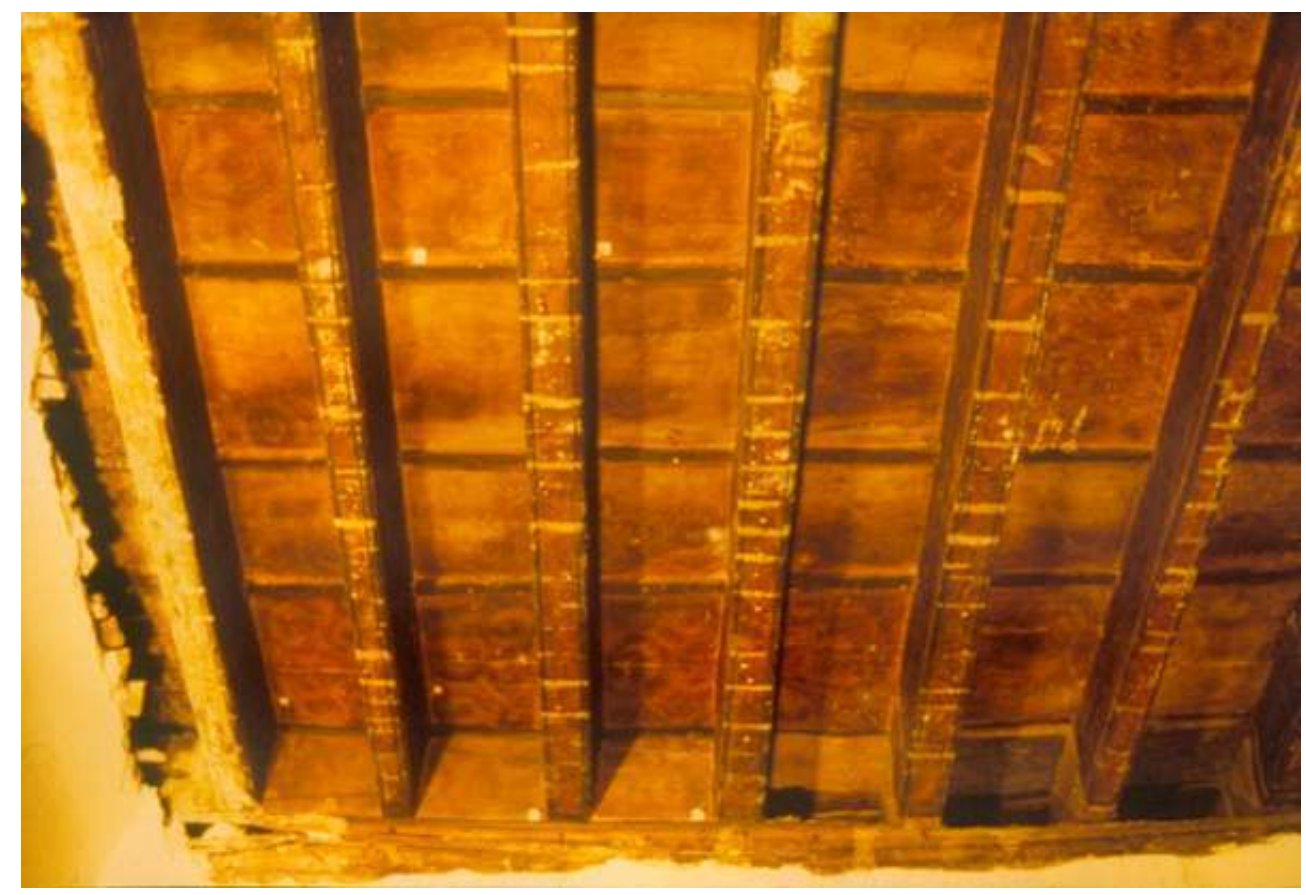

Auteur(s) : Mathieu, Véronique. Crédits : ADLFI - Mathieu, Véronique (2003)

Fig. $\mathrm{n}^{\circ} 3$ : Relevé d'état des lieux en plan du plafond

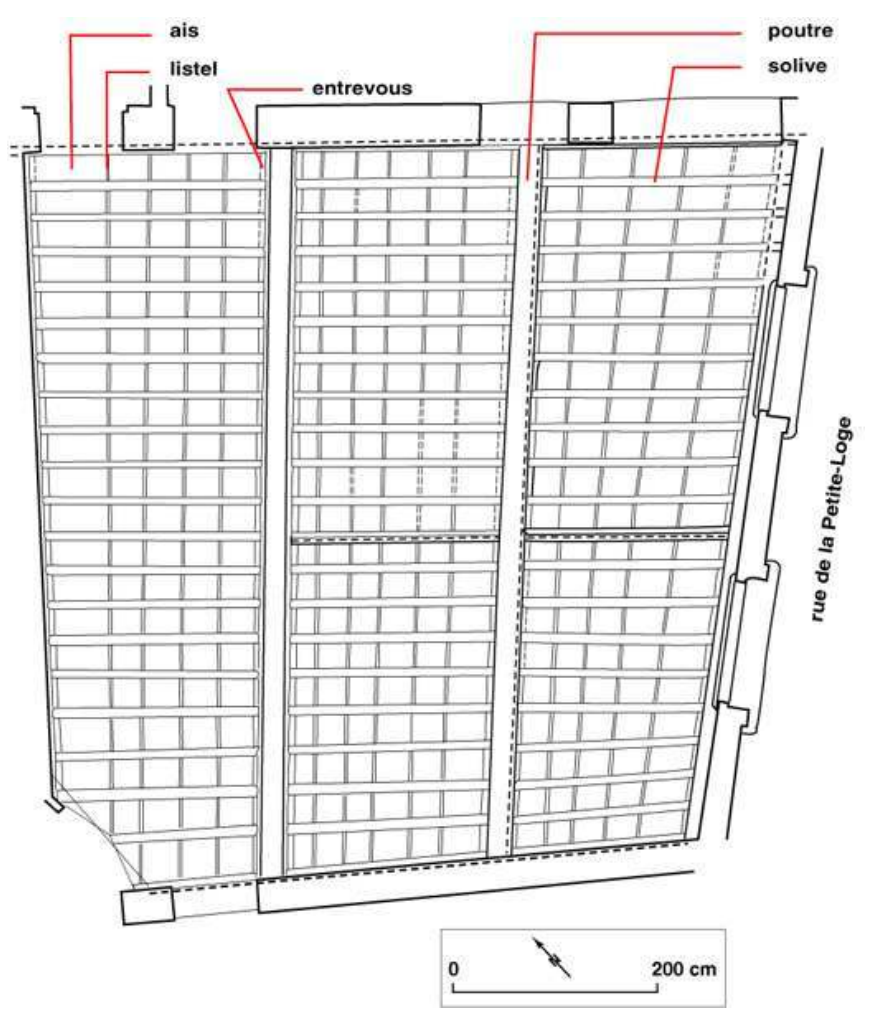

Auteur(s) : Mathieu, Véronique. Crédits : ADLFI - Mathieu, Véronique (2003) 
Fig. $n^{\circ} 4$ : Plan du plafond, liste et numérotation des poutres et solives

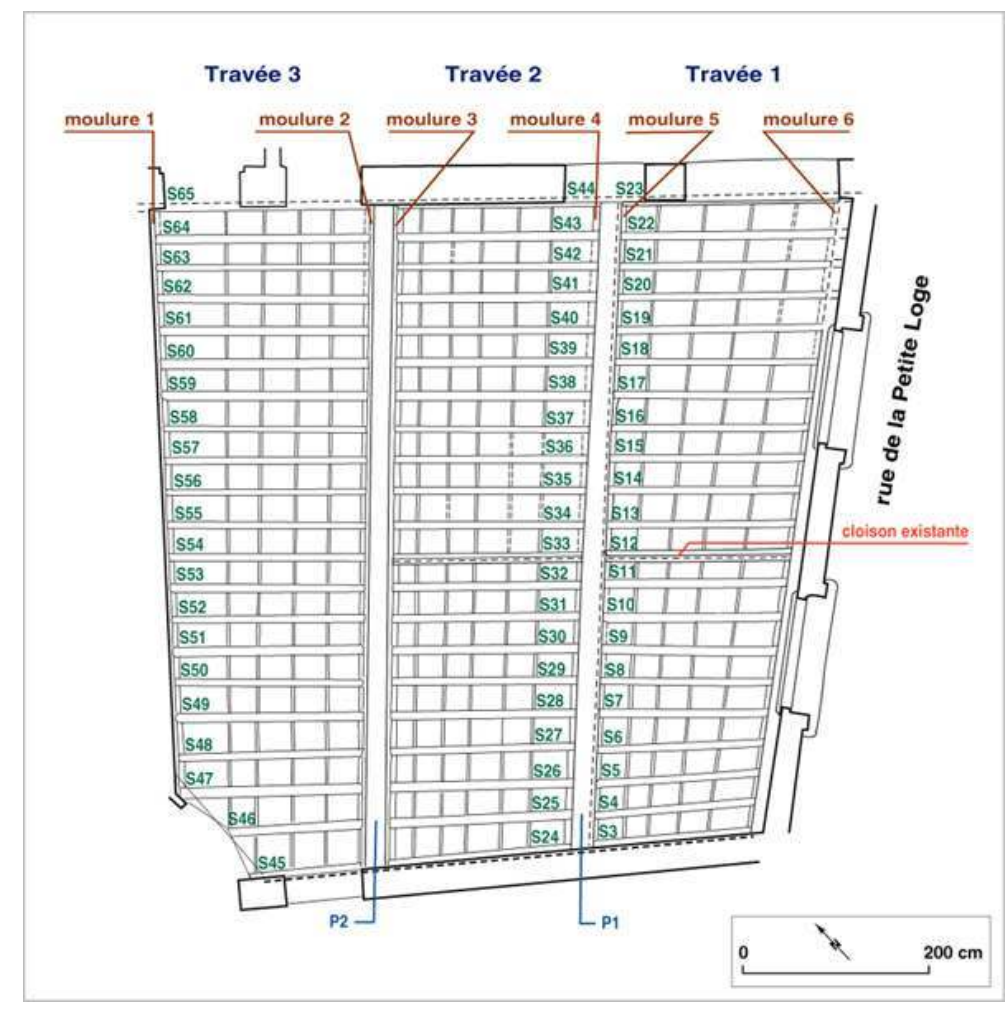

Auteur(s) : Mathieu, Véronique. Crédits : ADLFI - Mathieu, Véronique (2003)

Fig. $n^{\circ} 5$ : Plan du plafond, liste et numérotation des ais

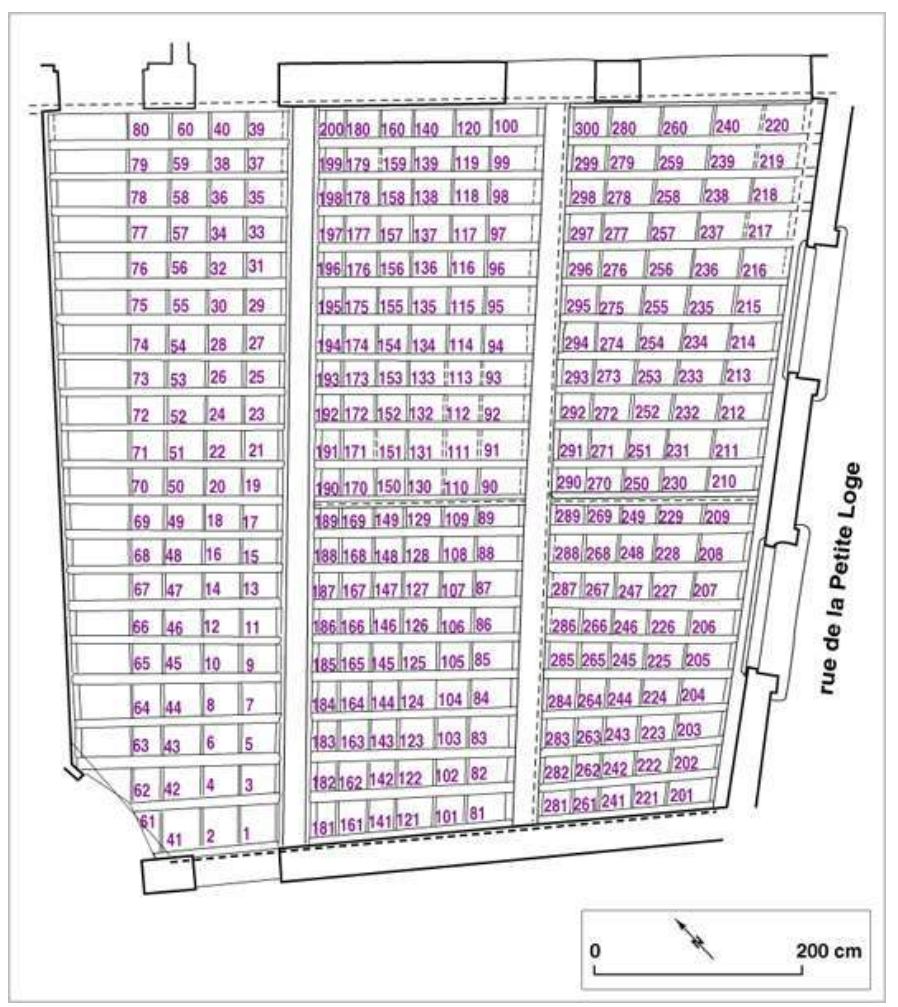

Auteur(s) : Mathieu, Véronique. Crédits : ADLFI - Mathieu, Véronique (2003) 
Fig. $n^{\circ} 6$ : Plan du plafond, repérage des coupe $A A$ et $B B$. Coupes $A A$ et $B B$

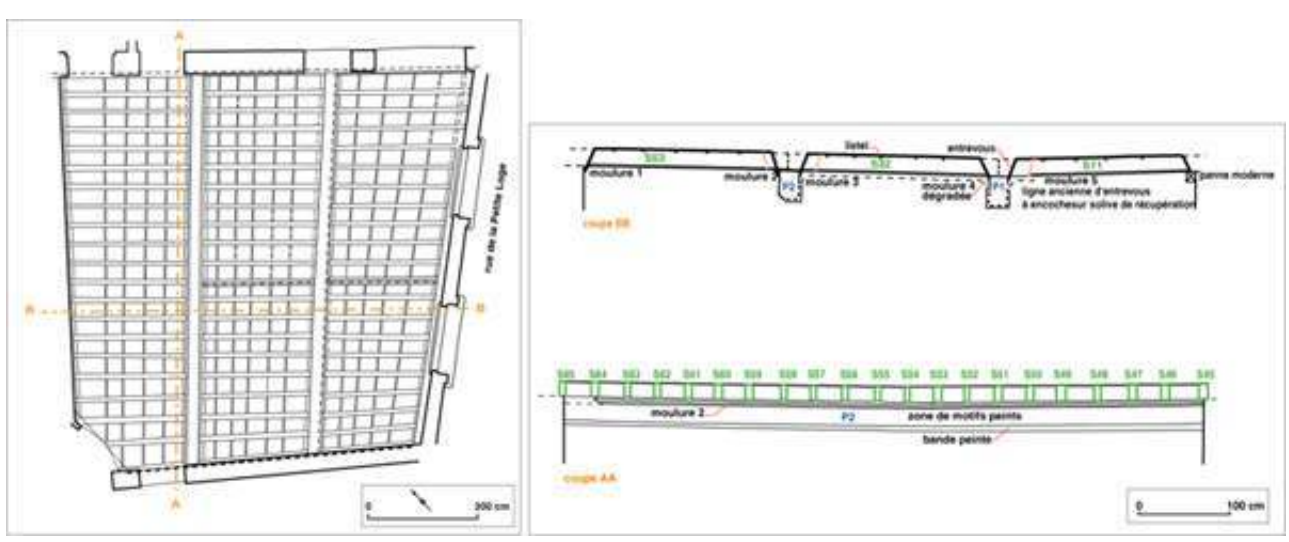

Auteur(s) : Mathieu, Véronique. Crédits : ADLFI - Mathieu, Véronique (2003)

Fig. $n^{\circ} 7$ : Détail du motif sur $P 2$, du motif ondé sur la baguette moulurée $n^{\circ} 2$, positionnement des clous, vue depuis le nord-ouest

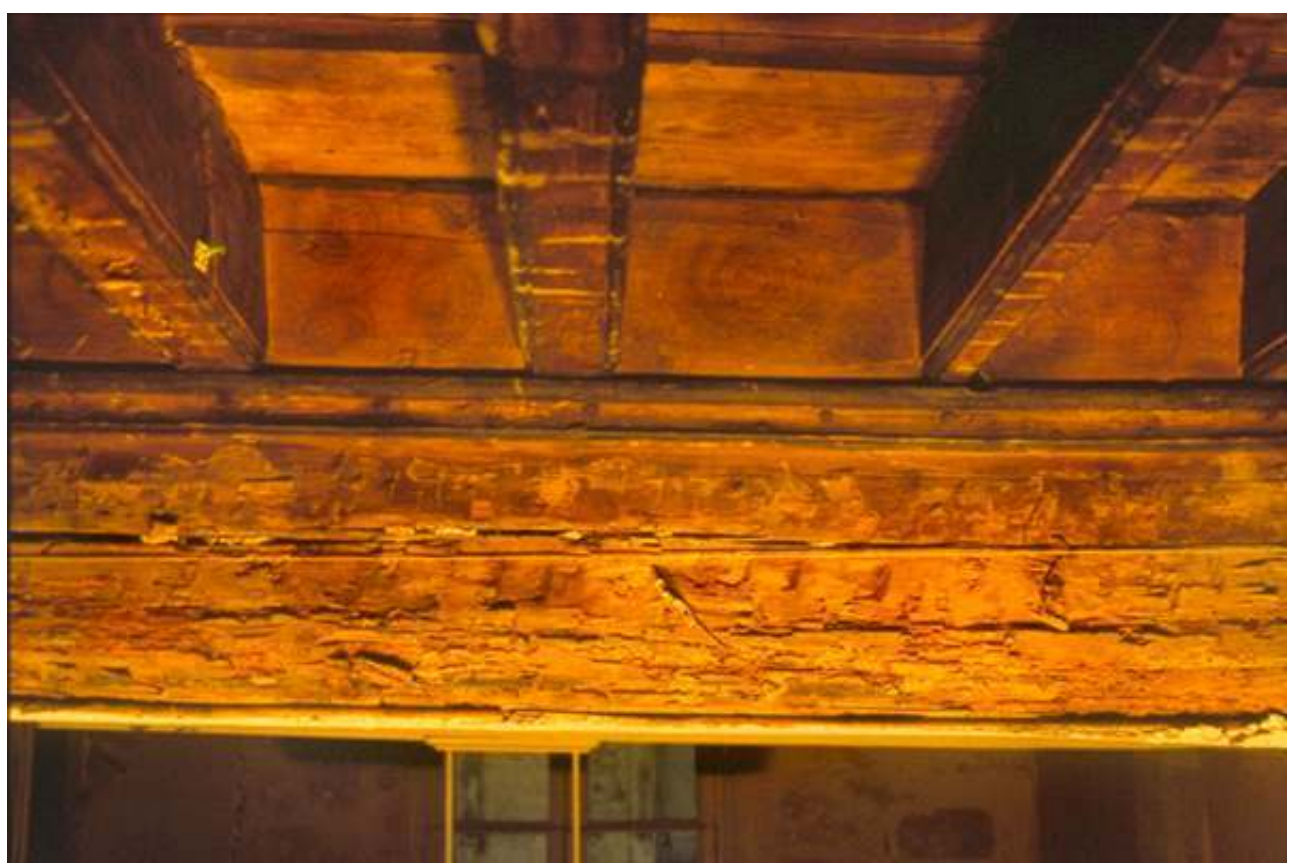

Auteur(s) : Mathieu, Véronique. Crédits : ADLFI - Mathieu, Véronique (2003) 
Fig. $n^{\circ} 8$ : Plan du plafond, repérage des sections sur solives et moulures

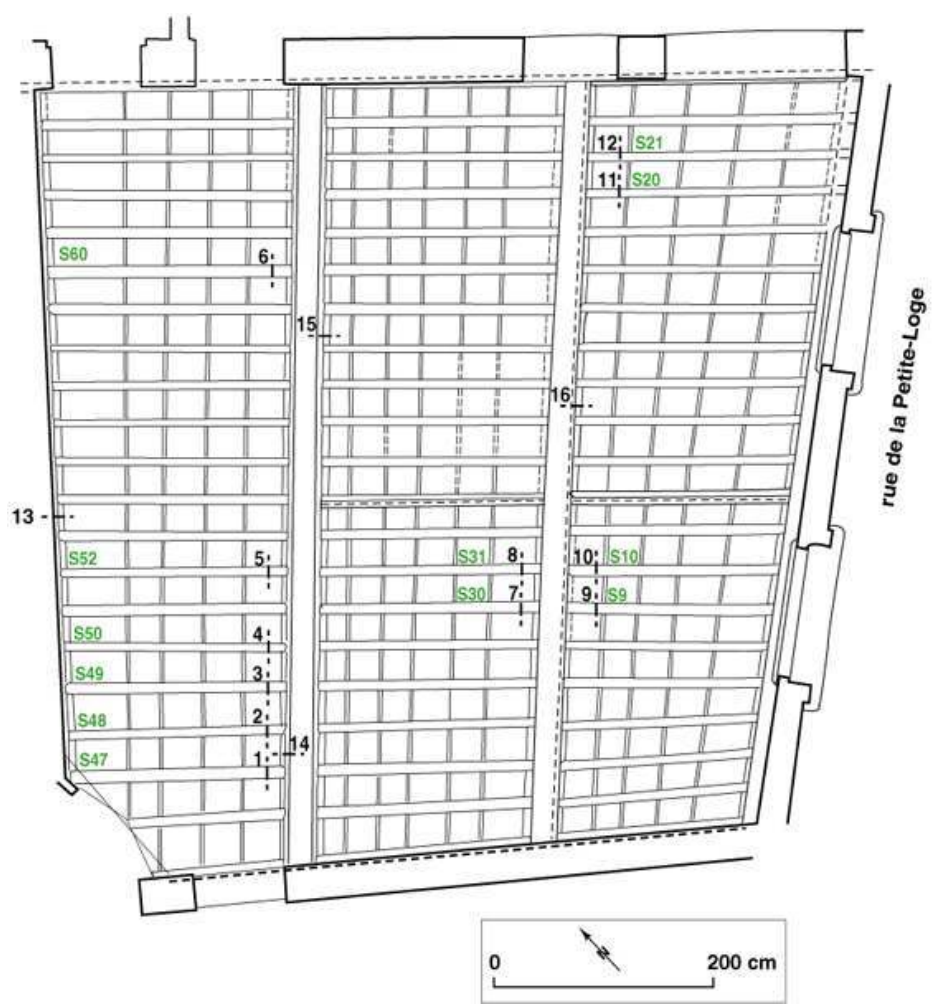

Auteur(s) : Mathieu, Véronique. Crédits : ADLFI - Mathieu, Véronique (2003)

Fig. $n^{\circ} 9$ : Coupes de détail sur les solives

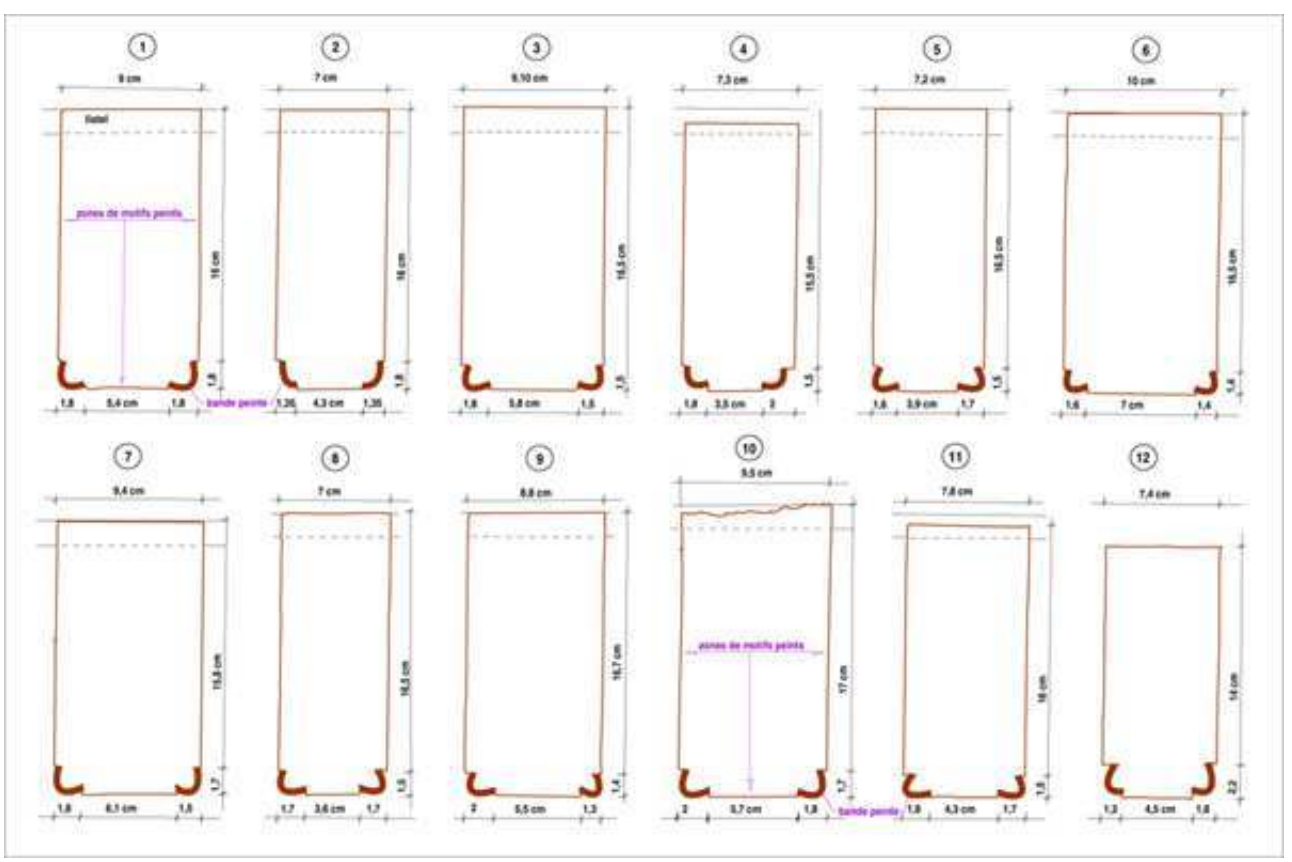

Auteur(s) : Mathieu, Véronique. Crédits : ADLFI - Mathieu, Véronique (2003) 
Fig. $\mathrm{n}^{\circ} 10$ : Coupes de détail sur les baguettes moulurées

(14)

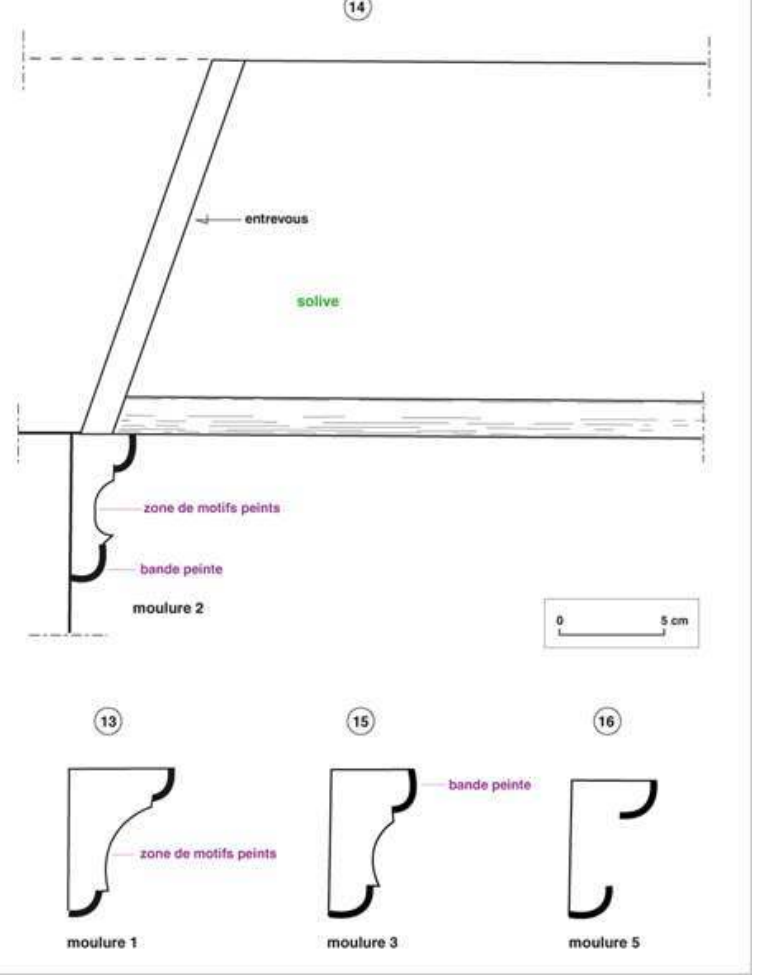

Auteur(s) : Mathieu, Véronique. Crédits : ADLFI - Mathieu, Véronique (2003)

Fig. $\mathrm{n}^{\circ} 11$ : Plan du plafond, repérage des solives de récupération et des solives filantes

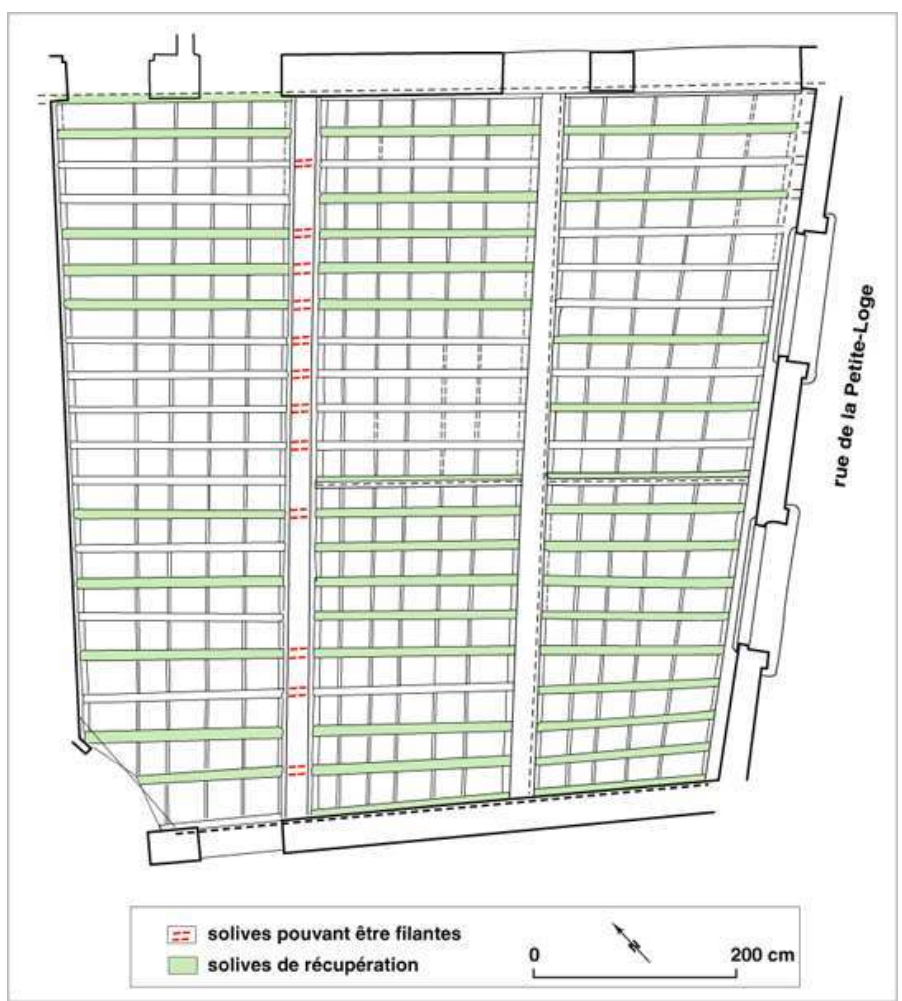

Auteur(s) : Mathieu, Véronique. Crédits : ADLFI - Mathieu, Véronique (2003) 
Fig. $\mathrm{n}^{\circ} 12$ : Détail du motif 22 sur joue de la solive $\mathrm{S} 60$, anciennes lignes d'entrevous, vus depuis le sud-ouest

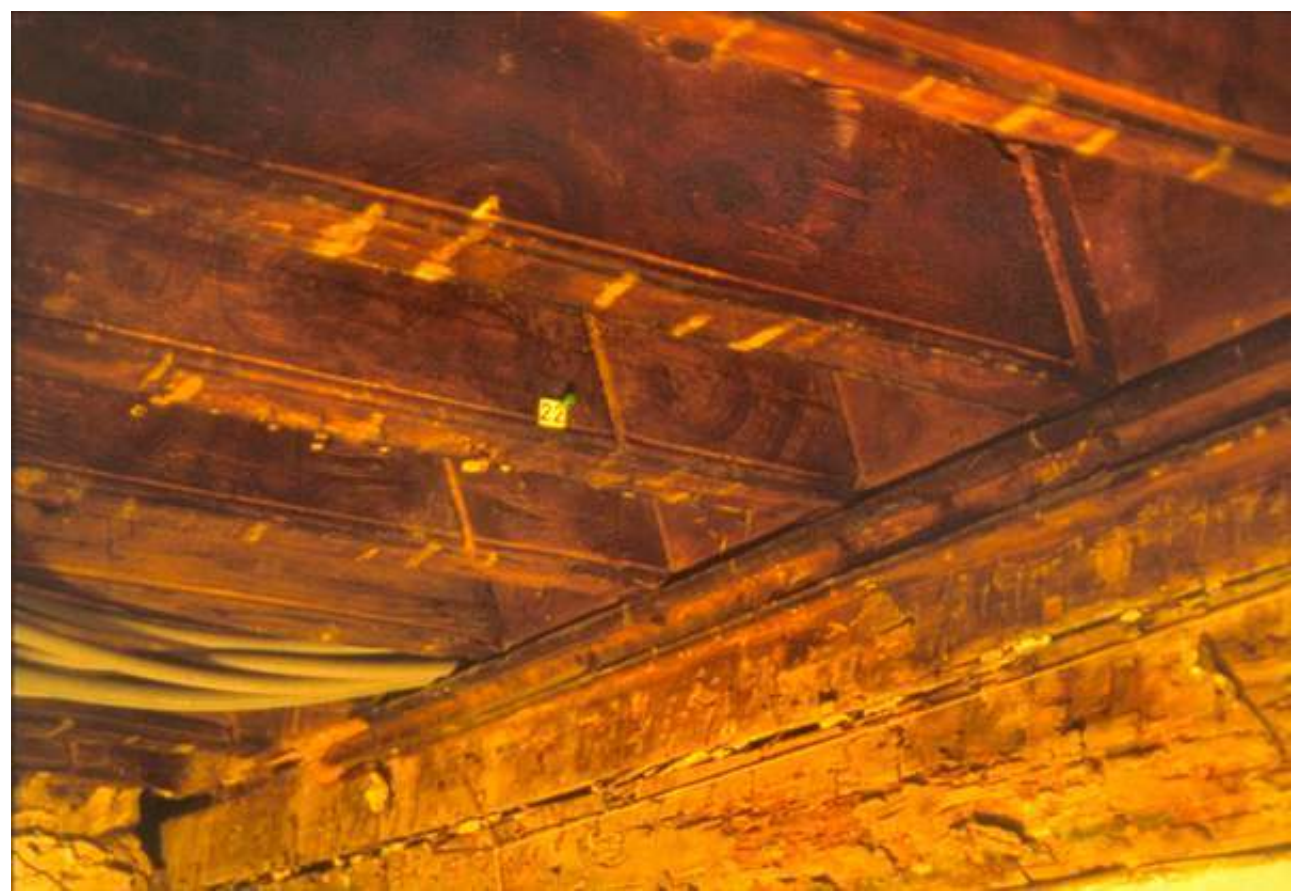

Auteur(s) : Mathieu, Véronique. Crédits : ADLFI - Mathieu, Véronique (2003)

Fig. $\mathrm{n}^{\circ} 13$ : Détail du motif 19 , visible sous la peinture au plomb, vu depuis le nord-est

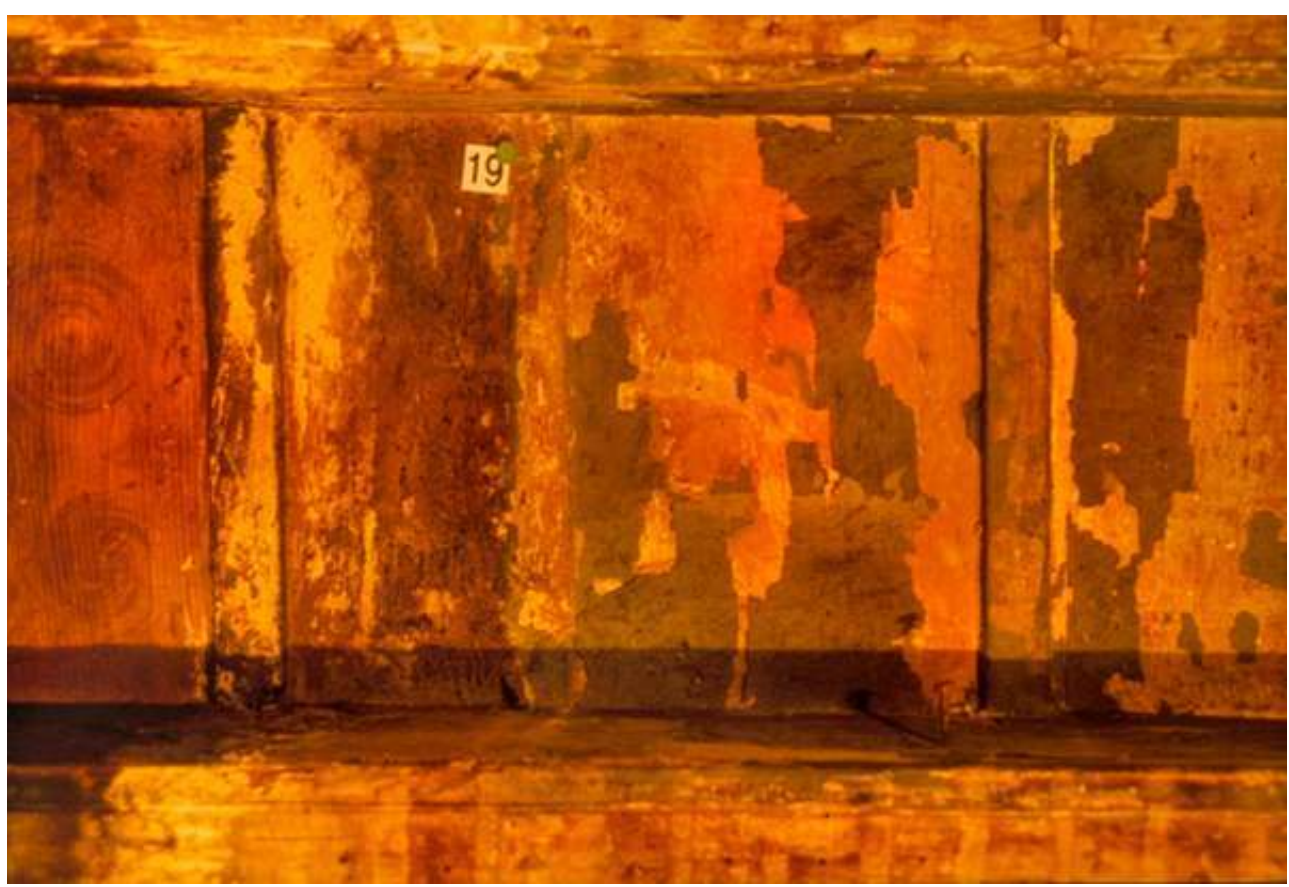

Auteur(s) : Mathieu, Véronique. Crédits : ADLFI - Mathieu, Véronique (2003) 
Fig. $\mathrm{n}^{\circ} 14$ : Détail des motifs 1 et 2 sur ais, détail sur motifs en sous-face des solives S48 et S49, vus depuis le sud-ouest

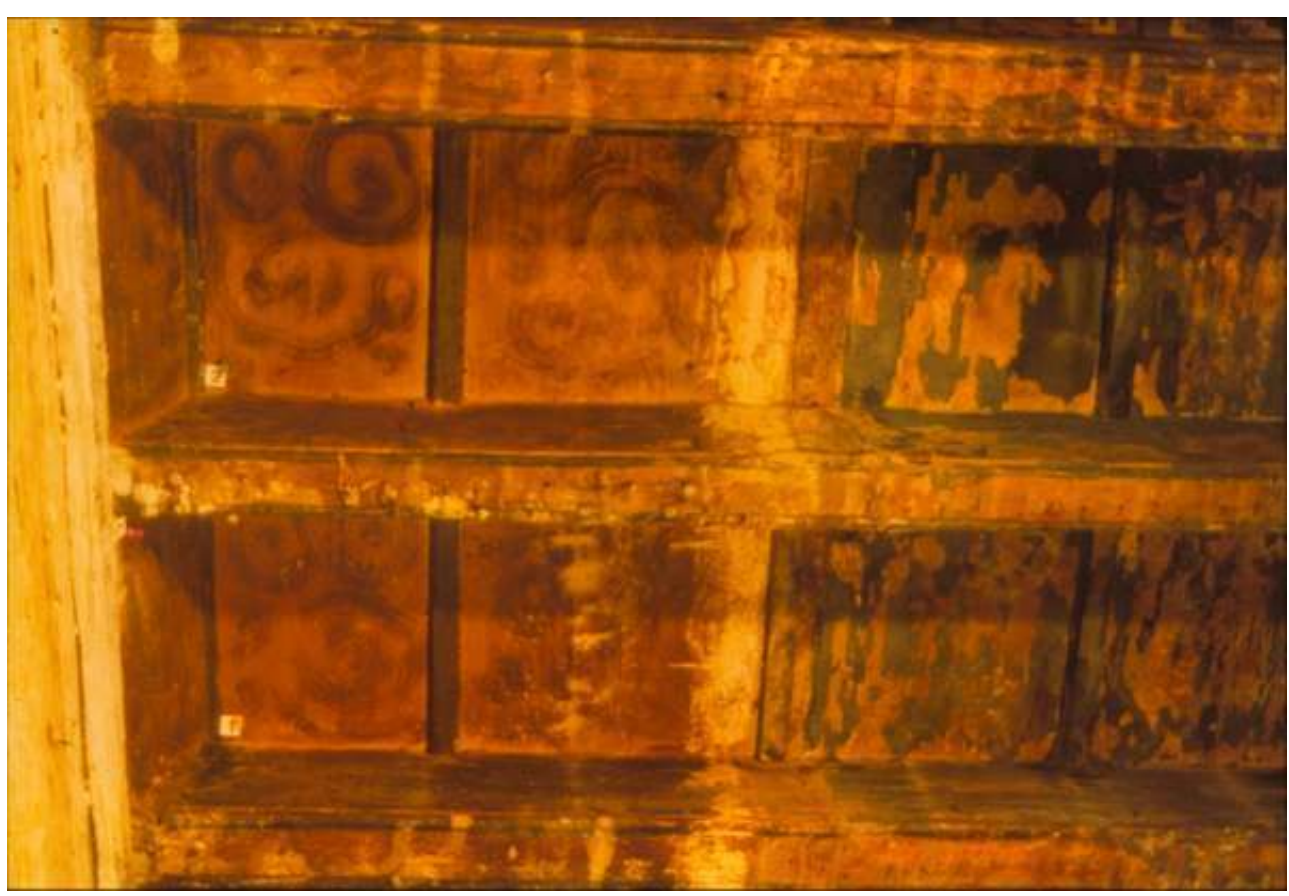

Auteur(s) : Mathieu, Véronique. Crédits : ADLFI - Mathieu, Véronique (2003)

Fig. $\mathrm{n}^{\circ} 15$ : Plan du plafond, zonage de l'état de conservation

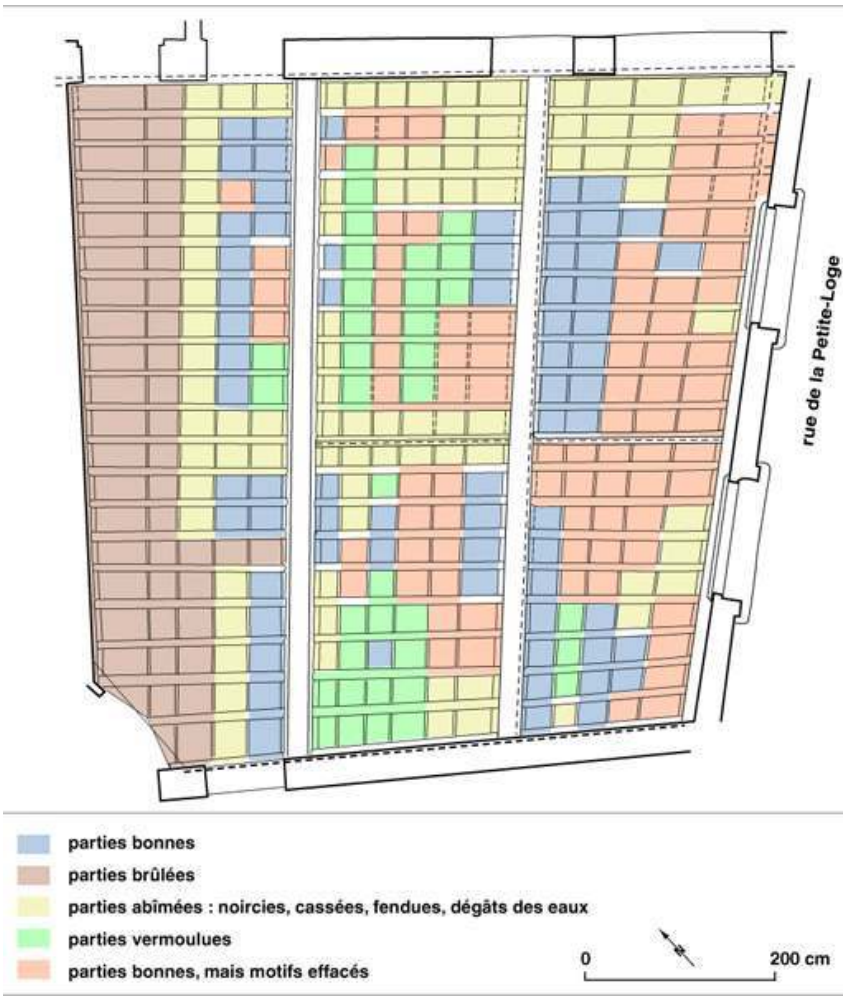

Auteur(s) : Mathieu, Véronique. Crédits : ADLFI - Mathieu, Véronique (2003) 
Fig. $\mathrm{n}^{\circ} 16$ : Détail des motifs 13 et 14 sur ais, vus depuis le nord-ouest

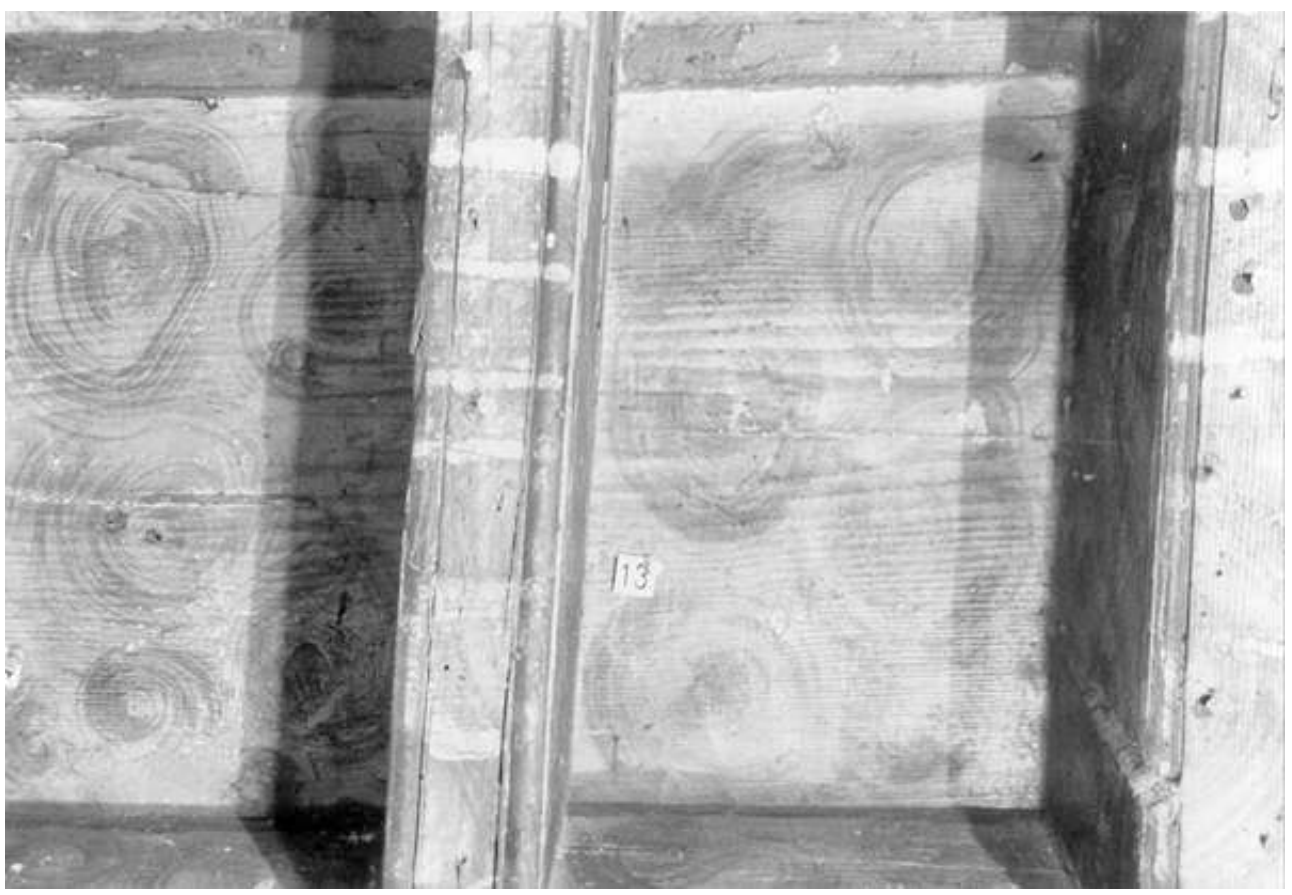

Auteur(s) : Mathieu, Véronique. Crédits : ADLFI - Mathieu, Véronique (2003)

Fig. $\mathrm{n}^{\circ} 17$ : Détail des motifs 17 et 18 sur ais, vus depuis le nord-ouest

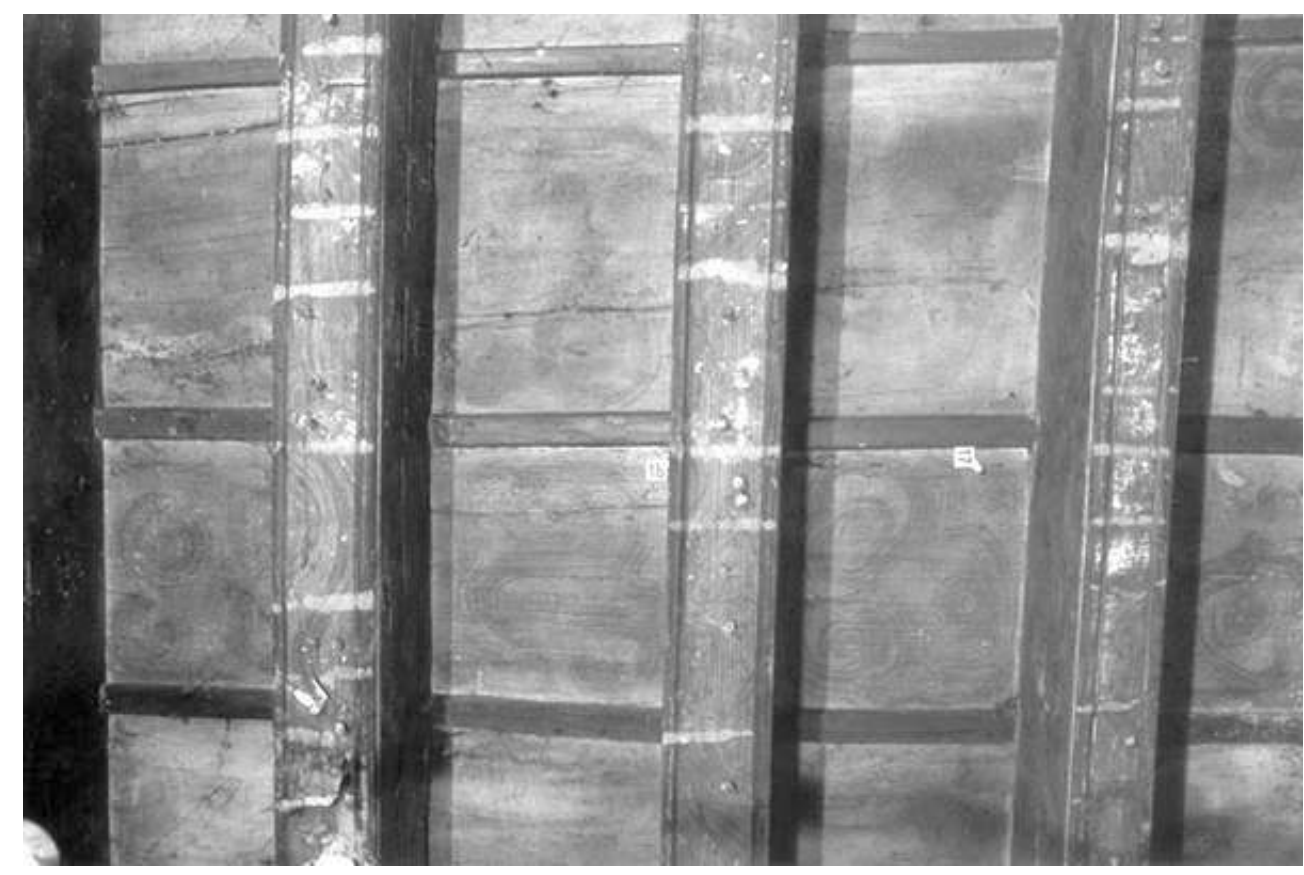

Auteur(s) : Mathieu, Véronique. Crédits : ADLFI - Mathieu, Véronique (2003) 
Fig. $n^{\circ} 18$ : Vue générale sur la troisième travée, de $S 58$ à $S 61$, vue depuis le nord-ouest

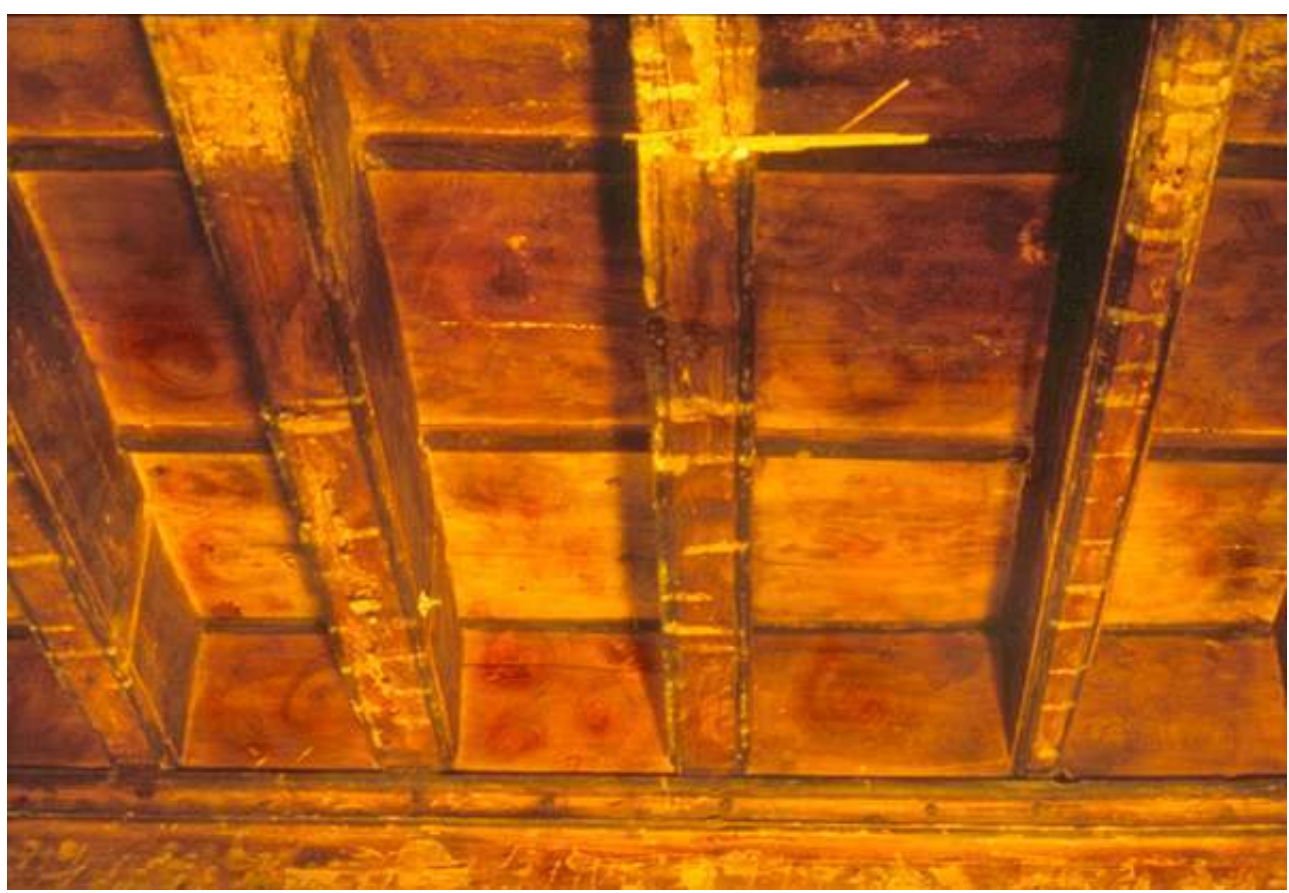

Auteur(s) : Mathieu, Véronique. Crédits : ADLFI - Mathieu, Véronique (2003)

Fig. $\mathrm{n}^{\circ} 19$ : Vue générale sur la première travée, de $\mathrm{S} 16$ à S20, vue depuis le sud-est

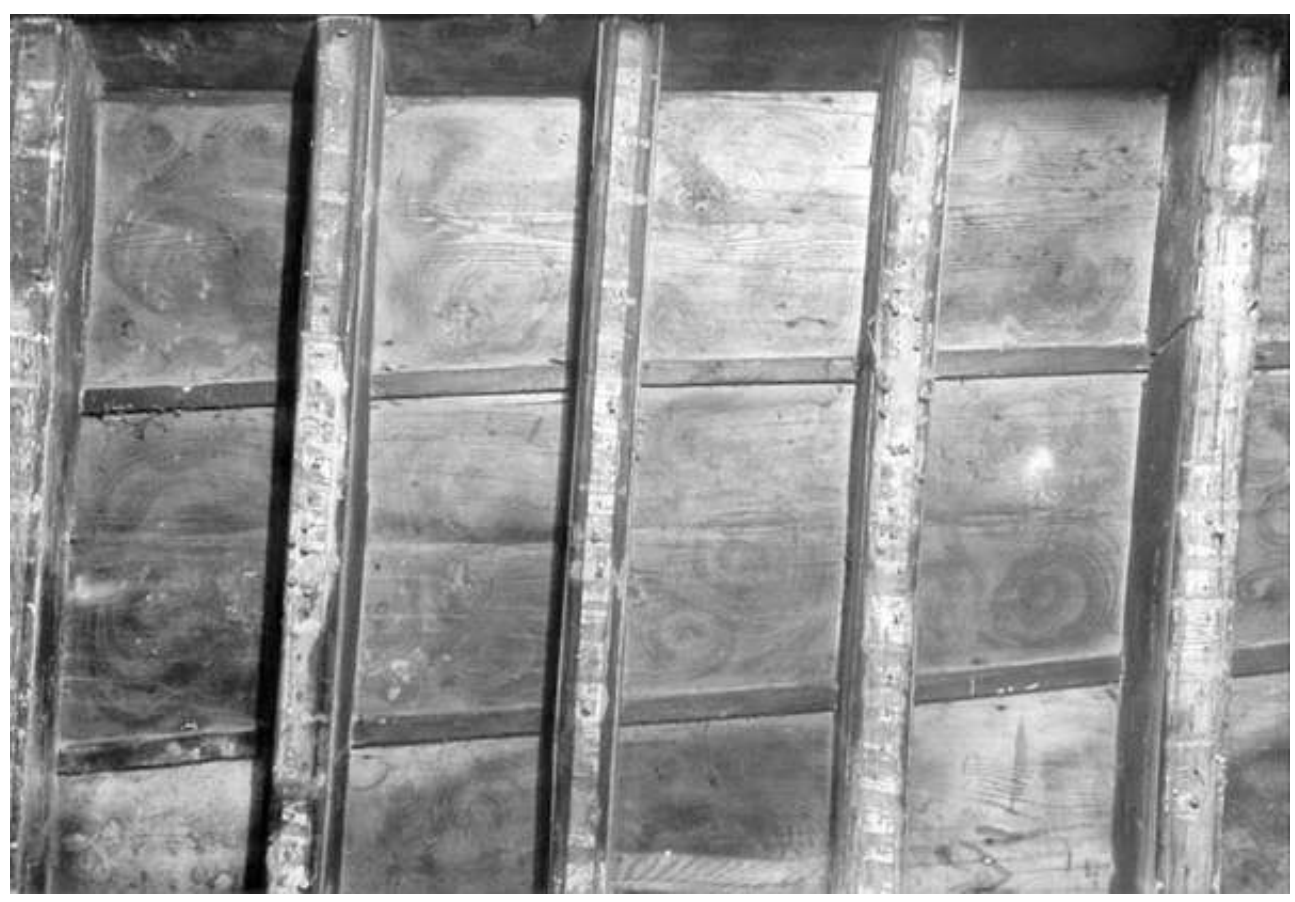

Auteur(s) : Mathieu, Véronique. Crédits : ADLFI - Mathieu, Véronique (2003) 
Fig. $n^{\circ} 20$ : Détail du motif 15 sur entrevous, vu depuis le nord-ouest

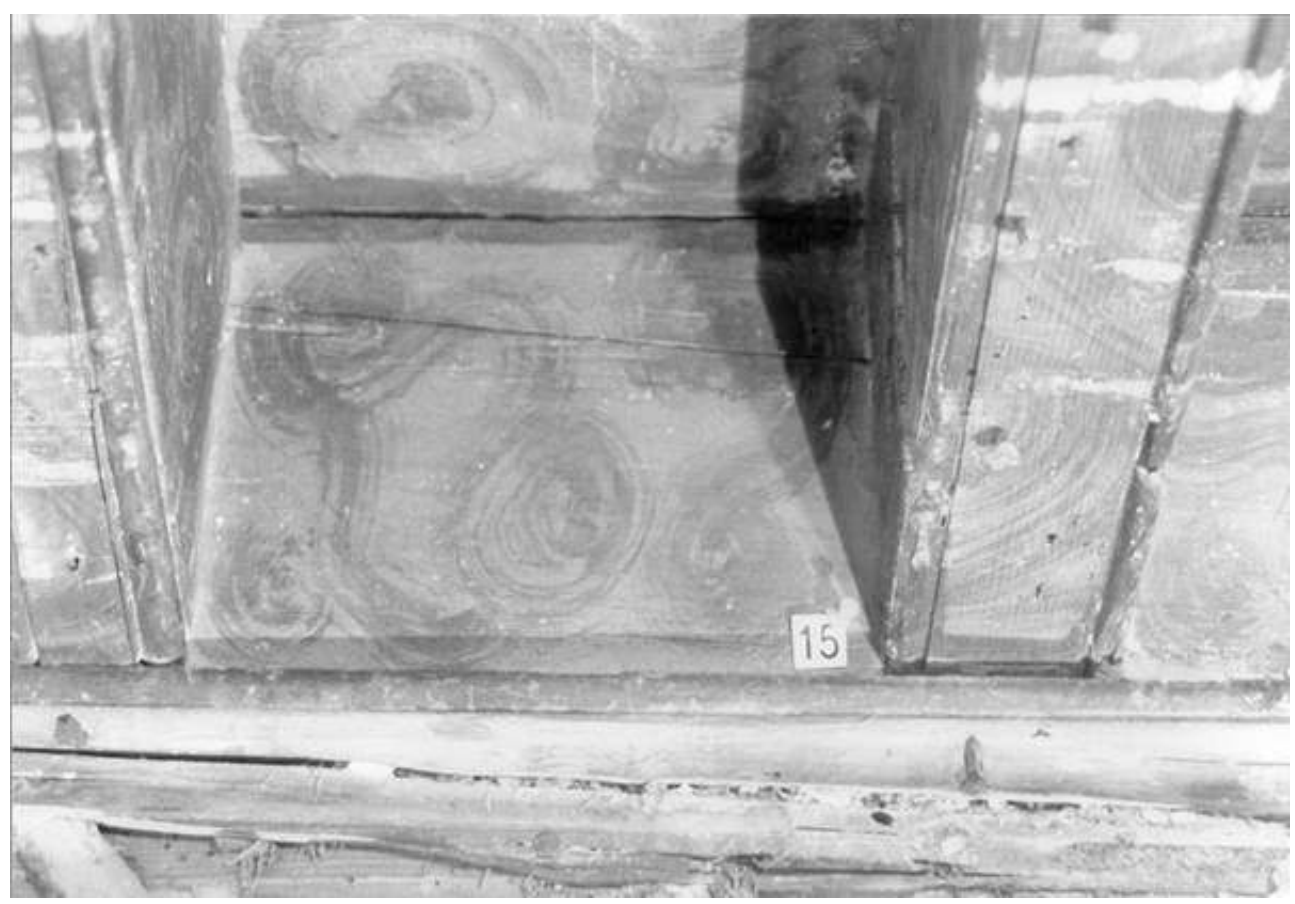

Auteur(s) : Mathieu, Véronique. Crédits : ADLFI - Mathieu, Véronique (2003)

Fig. $\mathrm{n}^{\circ} 21$ : Détail du motif 16 sur entrevous, vu depuis le nord-ouest

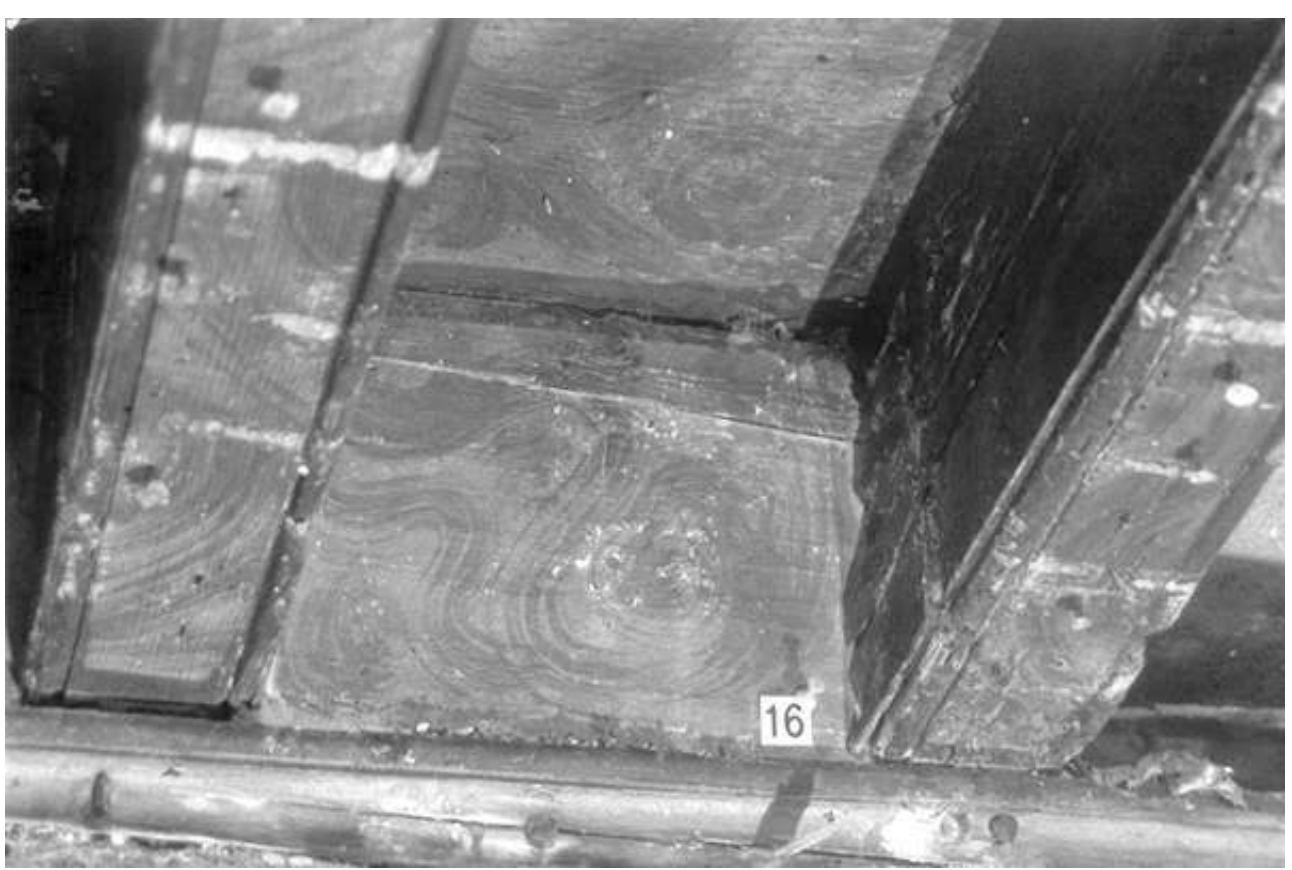

Auteur(s) : Mathieu, Véronique. Crédits : ADLFI - Mathieu, Véronique (2003) 
Fig. $\mathrm{n}^{\circ} 22$ : Détail du motif 21 sur $\mathrm{P} 2$, vu depuis le nord-ouest

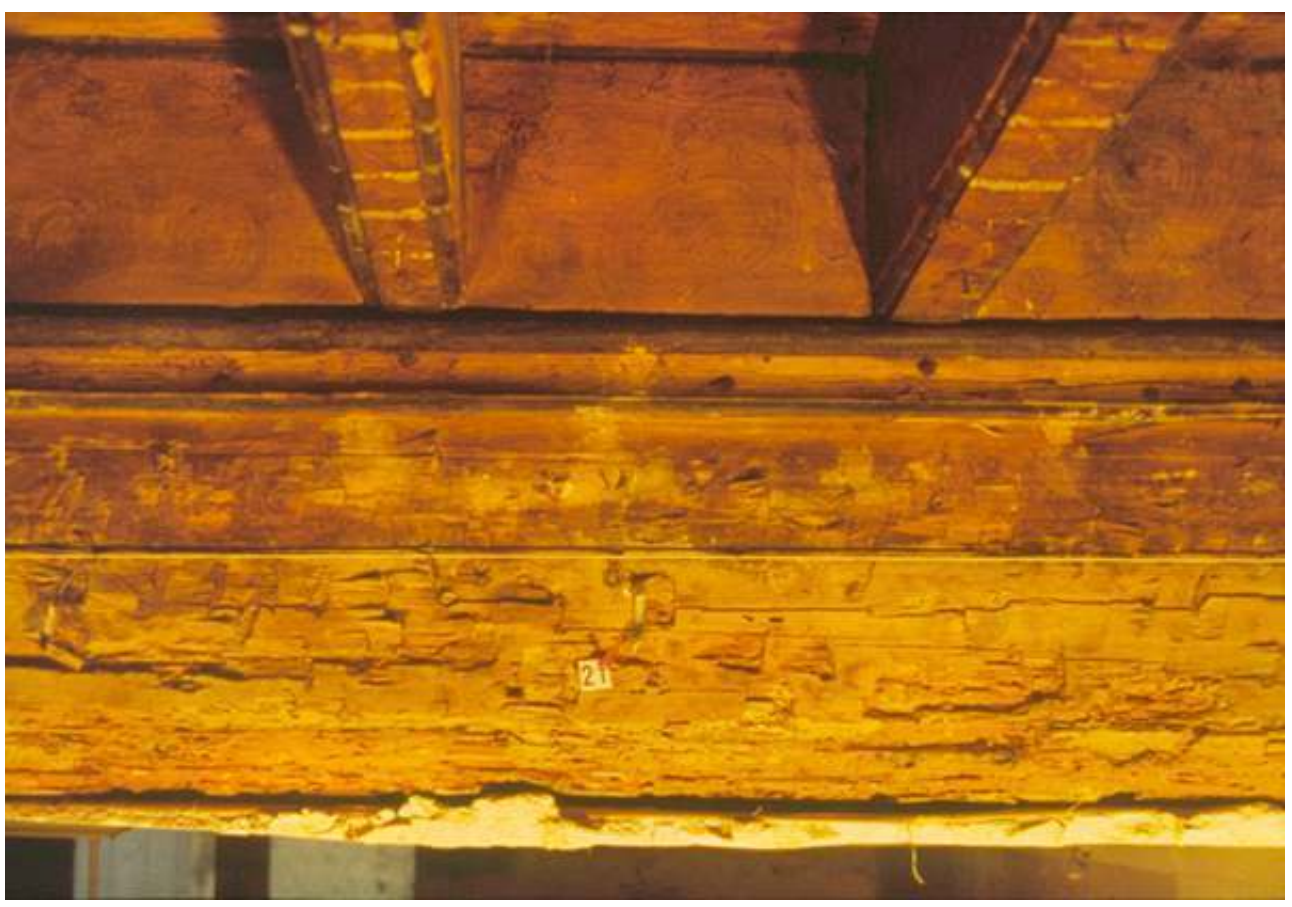

Auteur(s) : Mathieu, Véronique. Crédits : ADLFI - Mathieu, Véronique (2003)

Fig. $\mathrm{n}^{\circ} 23$ : Détail du motif 23 sur le mur entre $\mathrm{P} 1$ et $\mathrm{P} 2$, vu depuis le nord-est

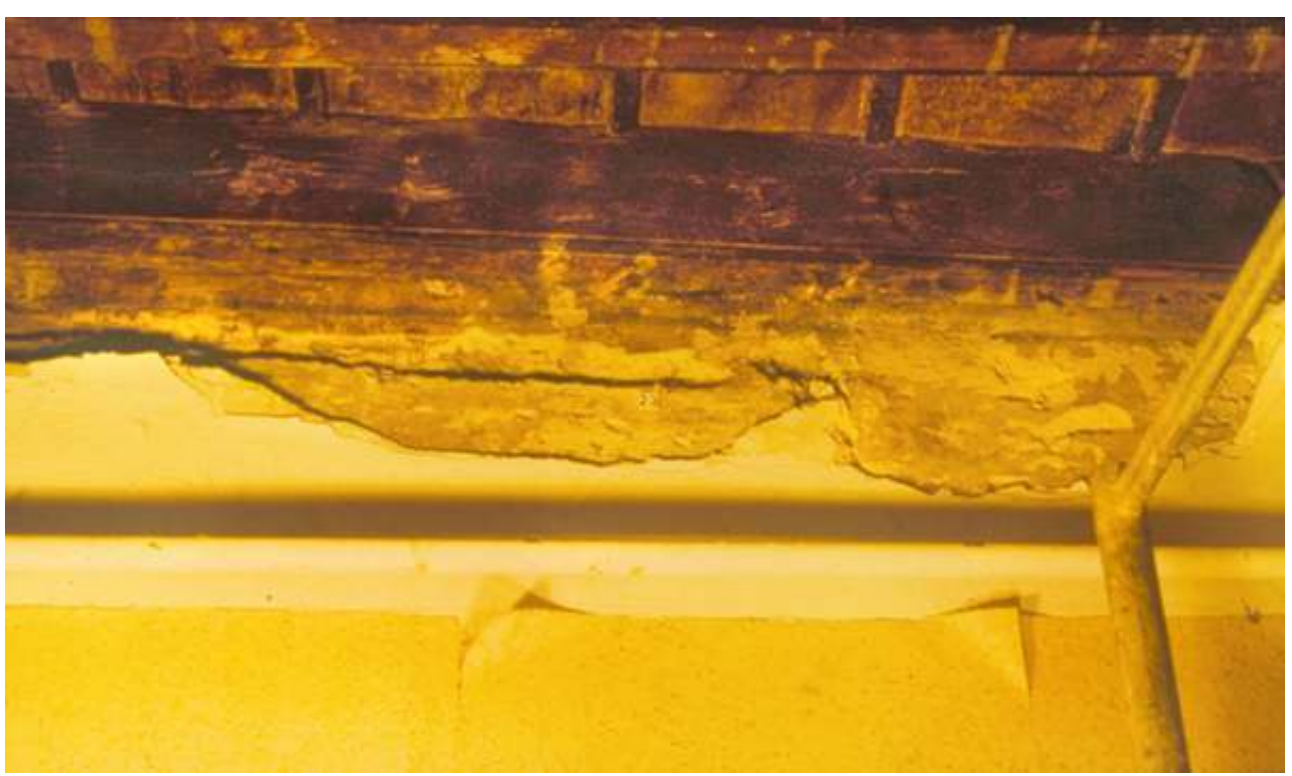

Auteur(s) : Mathieu, Véronique. Crédits : ADLFI - Mathieu, Véronique (2003) 
Fig. $\mathrm{n}^{\circ} 24$ : Plan du plafond, repérage des prises de vues

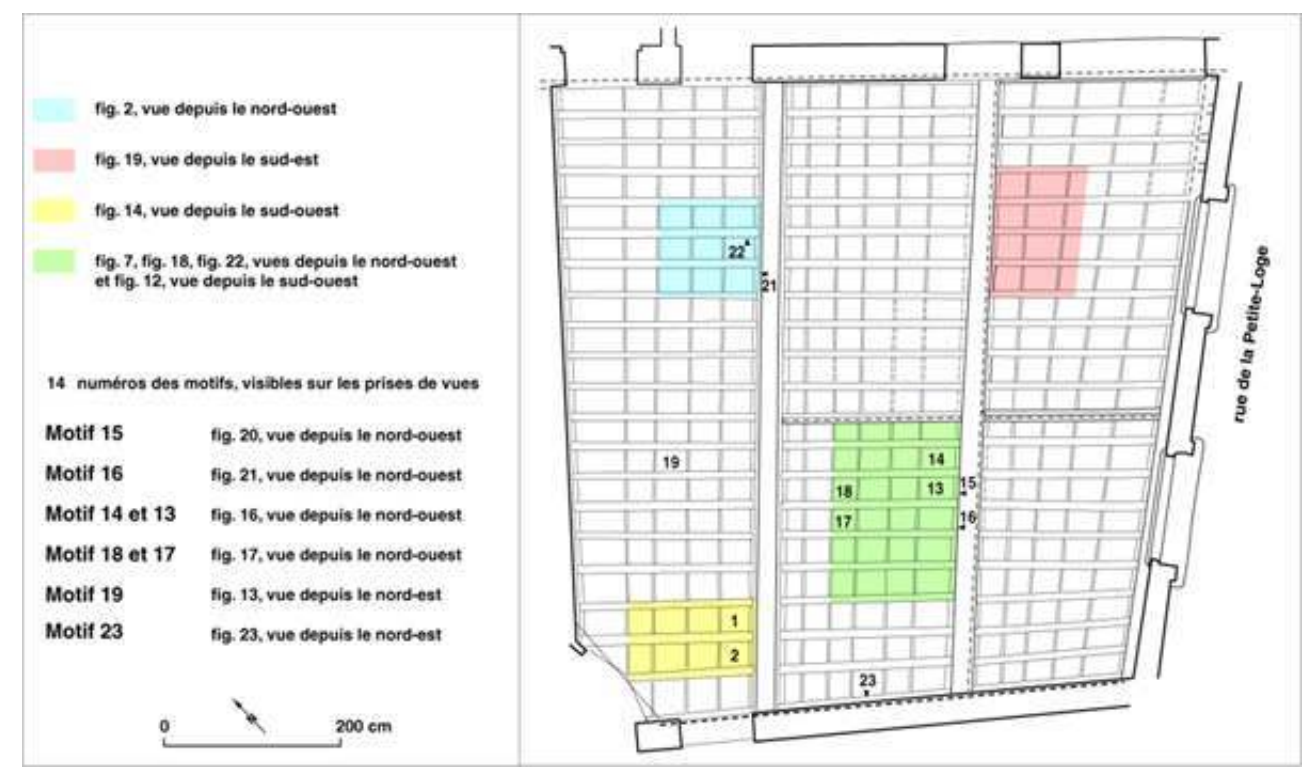

Auteur(s) : Mathieu, Véronique. Crédits : ADLFI - Mathieu, Véronique (2003)

\section{INDEX}

Index chronologique : ép médiévale, Temps Modernes

Index géographique : Languedoc-Roussillon, Hérault, Montpellier operation sauvetage urgent (SU)

\section{AUTEURS}

\section{VÉRONIQUE MATHIEU}

AFAN 